\title{
Self-Localized Quasi-Particle Excitation in Quantum Electrodynamics and Its Physical Interpretation ${ }^{\star}$
}

\author{
Ilya D. FERANCHUK and Sergey I. FERANCHUK
}

Department of Physics, Belarusian University, 4 Nezavisimosti Ave., 220030, Minsk, Belarus

E-mail:fer@open.by,sergey@feranchuk.linux.by

URL: http://www. theorphysics.bsu.by/Stuff/Feranchuk.htm, http://sergey.feranchuk.net

Received October 21, 2007, in final form November 29, 2007; Published online December 07, 2007

Original article is available at http://www.emis.de/journals/SIGMA/2007/117/

\begin{abstract}
The self-localized quasi-particle excitation of the electron-positron field (EPF) is found for the first time in the framework of a standard form of the quantum electrodynamics. This state is interpreted as the "physical" electron (positron) and it allows one to solve the following problems: i) to express the "primary" charge $e_{0}$ and the mass $m_{0}$ of the "bare" electron in terms of the observed values of $e$ and $m$ of the "physical" electron without any infinite parameters and by essentially nonperturbative way; ii) to consider $\mu$-meson as another self-localized EPF state and to estimate the ratio $m_{\mu} / m$; iii) to prove that the selflocalized state is Lorentz-invariant and its energy spectrum corresponds to the relativistic free particle with the observed mass $m$; iv) to show that the expansion in a power of the observed charge $e \ll 1$ corresponds to the strong coupling expansion in a power of the "primary" charge $e_{0}^{-1} \sim e$ when the interaction between the "physical" electron and the transverse electromagnetic field is considered by means of the perturbation theory and all terms of this series are free from the ultraviolet divergence.
\end{abstract}

Key words: renormalization; Dirac electron-positron vacuum; nonperturbative theory

2000 Mathematics Subject Classification: 81V05; 81V10; 83C47

\section{Introduction}

It is no doubt at present that the Standard Model is the fundamental basis for the theory of the electro-weak interaction [1]. It means that the quantum electrodynamics (QED) is actually the part of the general gauge theory. Nevertheless, QED considered by itself as the isolated system remains the most successful quantum field model that allows one to calculate the observed characteristics of the electromagnetic processes with a unique accuracy (for example, [2, 3]). It is well known that these calculations are based on the series of rules connected with the perturbation theory in the observed charge $e$ of the "physical" electron and the renormalization property of QED. The latter one means that the "primary" parameters of the theory (the charge $e_{0}$ and the mass $m_{0}$ of the "bare" electron) that are defined by the divergent integrals, can be excluded from the observed values. However, even the creators of the present form of QED were not satisfied because "the calculation rules of QED are badly adjusted with the logical foundations of quantum mechanics and they cannot be considered as the satisfactory solution of the difficulties" [4, $\S 81]$ and "it is simply a way to sweep the difficulties under the rug" [5].

There are a number motivations for calculation of the "bare" electron characteristics $e_{0}$ and $m_{0}$ in spite the fact that these values are unobserved. First of all it is the question whether

${ }^{\star}$ This paper is a contribution to the Proceedings of the Seventh International Conference "Symmetry in Nonlinear Mathematical Physics" (June 24-30, 2007, Kyiv, Ukraine). The full collection is available at http://www.emis.de/journals/SIGMA/symmetry2007.html 
the system of Maxwell and Dirac equations as the mathematical model for the quantum field system is closed and self-consistent when considering the processes related to the interaction of electrons, positrons and photons? In that sense QED in the existing form of renormalization is an unclosed theory because it includes an additional, external dimensional parameter which enables the regularization of integrals. The contradiction between the small value of the coupling constant and its infinite calculated value shows a logical inconsistency of the perturbation theory in QED, so called "Landau pole" [6, $\S 128]$. It is very important to consider the renormalization problem in the framework of the "logical principles of quantum mechanics" in order to develop some new nonperturbative approach in the field theory in application to the real physical system with completely defined Hamiltonian. At present nonperturbative methods are mainly studied for quite abstract quantum field models with a strong interaction (for example, [7]). Such methods may be especially interesting for the non-renormalized quantum field models.

It is also very essential that the dynamical description of the internal structure of the "physical" electron gives the fundamental possibility to consider $\mu$-meson as an excited state of the electron-positron field as it has been shown by Dirac [8].

The relation between the "primary" coupling constant $e_{0}$ and the charge $e$ is undetermined in the present form of QED. Therefore it is possible that the value $e_{0}$ is large in spite the observed renormalized charge being small $e \ll 1$. Precisely this possibility $\left(e_{0}>1\right.$, but $\left.e \ll 1\right)$ is investigated in the present paper in order to find a spectrum of the quasi-particle excitations in QED without the perturbation theory. Our main goal is to find such a form of the renormalization that would be logically consistent but the calculation possibilities of QED for the observed values would be preserved.

It is important to stress that the canonical QED model is considered with a nonzero mass of the "bare" electron $m_{0} \neq 0$ and without the chiral symmetry. This approach is distinguished essentially from the nonperturbative analysis of the strong coupling QED model with a zero mass of the fermion field (for example, $[9,10]$ and citation therein). In the latter case the observed particle mass appears as the result of spontaneous breaking of the chiral symmetry, however, the cut-off momentum $L$ is another undefined parameter in such theories.

The article is structured in the following way. In Section 2 it is shown for the first time that the self-localized one-particle excitation can be found in the spectrum of QED Hamiltonian. This state cannot be calculated by means of the perturbation theory as a power series of the coupling constant $e_{0}$. The stability of this state is conditioned by a localized charge distribution of the electron-positron field coupled with a scalar component of the electromagnetic field. The system of nonlinear equations for these spatially localized distributions is derived and its numerical solution is obtained.

In Section 3 the self-localized excitation is interpreted as the "physical" electron with the observed values of the charge $e$ and mass $m$. It allowed us to express characteristics $e_{0}$ and $m_{0}$ of the "bare" electron that are actually the parameters of the initial Hamiltonian in terms of $e$ and $m$. It is shown that the relation between these values includes the singularity in the limit of $e \rightarrow 0$ and cannot be calculated by means of the perturbation theory. This result cannot be also obtained in the framework of the "quenched QED" model based on the Schwinger-Dyson equation [11] or on the variational approach [12] because the charge renormalization did not take into account in this model.

The considered physical interpretation of the self-localized state leads also to an important consequence. It is shown in Section 3 that there is another one-particle excitation of the electronpositron field with the same charge and spin as for electron but with the larger mass. Following Dirac [8] this excitation was considered as the "physical" $\mu$-meson and the ratio $m / m_{\mu}$ is calculated. The calculated mass of $\mu$-meson proved to be very close to its experimental value. It is essential that unlike [8] the $\mu$-meson mass is calculated without any additional parameters of the model with the exception of $e_{0}$ and $m_{0}$. 
The localized charge distribution in the "physical" electron corresponds to the spontaneous Lorentz symmetry breaking for the considered system. This phenomenon is typical for the quantum field theories with the particle-field strong coupling (for example, the "polaron" problem $[13,14])$. It is shown in Section 4 that reconstruction of this symmetry leads to the dependence of energy of the excitation on its total momentum. It is essential that this dependence corresponds exactly the relativistic kinematics of a free particle with the observed mass $m$.

It is shown in Section 5 that the strong coupling series in the initial QED Hamiltonian corresponds to the perturbation theory in terms of observed charge $e \sim e_{0}^{-1} \ll 1$. However, all high-order corrections of this perturbation theory is defined by the convergent integrals without any additional cut-off parameters. The correspondence of these results with the standard renormalization procedure when calculating the observed characteristics of the electromagnetic processes is also discussed.

\section{Self-localized state with zero momentum}

It is well known that the spatially localized states are very important for a lot of quantum field models. Let us remind the one-dimensional model for the scalar field with the Hamiltonian (for example, [15]):

$$
\hat{H}=\int d x \frac{1}{2}\left\{\hat{\pi}^{2}+\left(\frac{\partial \hat{\varphi}(x)}{\partial x}\right)^{2}+\frac{1}{2} \lambda\left(\hat{\varphi}(x)^{2}-\frac{m^{2}}{\lambda}\right)^{2}\right\},
$$

with the field operators $\hat{\varphi}(x)$ and the corresponding momentum operators $\hat{\pi}$.

This Hamiltonian has the eigenvector $|\varphi\rangle$ with the energy density localized in the vicinity of an arbitrary point $x_{0}$. With the zero total momentum of the system the energy density is distributed as follows:

$$
E(x)=\frac{m^{4}}{2 \lambda} \cosh ^{-4}\left[\frac{m}{\sqrt{2}}\left(x-x_{0}\right)\right],
$$

and corresponds to the finite total energy $E_{0}=(2 \sqrt{2} / 3)\left(m^{3} / \lambda\right)$. Lorentz-invariance of the system leads to the relativistic dependence of the energy on the momentum of the localized excitation [15].

It is important for the further discussion that the state (1) cannot be derived by means of the perturbation theory based on the coupling constant $\lambda$ connected with the nonlinear interaction. One should separate the non zero classical component from the field operators $\varphi(x)=\langle\varphi|\hat{\varphi}| \varphi\rangle$ averaged over the considered state even in the zeroth-order approximation. And the classical function $\varphi(x)$ is not reduced to the plane wave as in the zeroth-order perturbation theory. It is satisfied to the nonlinear differential equation that is defined by the variation of the classical functional.

The Fröhlich model for "polaron" problem gives another example [16]. This model corresponds to the electron-phonon interaction in the ionic crystals and is described by the following Hamiltonian:

$$
\hat{H}=\frac{1}{2} \hat{p}^{2}+\sum_{\vec{k}} a_{\vec{k}}^{+} a_{\vec{k}}+2^{3 / 4} \sqrt{\frac{\pi \alpha}{\Omega}} \sum_{\vec{k}} \frac{1}{k}\left(a_{\vec{k}}+a_{-\vec{k}}^{+}\right) e^{i \vec{k} \cdot \vec{r}} .
$$

In this case the spatially localized electron state ("polaron") cannot be found also by means of the perturbation theory in terms of the electron-phonon coupling constant $\alpha$. The variational wave function of the electron and the classical part of the phonon field $u_{\vec{k}}=\left\langle a_{\vec{k}}\right\rangle$ should be calculated even in the zeroth-order approximation. The translational symmetry of the initial 
Hamiltonian leads to the dependence of the energy of this one-particle excitation on its total momentum [14].

Let us now consider the nonperturbative analysis of the spectrum of the one-particle excitations of the QED Hamiltonian that is defined by the following form (for example, [17]):

$$
\begin{aligned}
& \hat{H}=\int d \vec{r}:\left\{\hat{\psi}^{*}(\vec{r})\left[\vec{\alpha}\left(\vec{p}+e_{0} \hat{\vec{A}}(\vec{r})\right)+\beta m_{0}\right] \hat{\psi}(\vec{r})+e_{0} \hat{\varphi}(\vec{r}) \hat{\rho}(\vec{r})-\frac{1}{2}(\vec{\nabla} \hat{\varphi}(\vec{r}))^{2}\right\}:+\sum_{\vec{k} \lambda} \omega(\vec{k}) \hat{n}_{\vec{k} \lambda}, \\
& \hat{\rho}(\vec{r})=\frac{1}{2}\left[\hat{\psi}^{*}(\vec{r}) \hat{\psi}(\vec{r})-\hat{\psi}(\vec{r}) \hat{\psi}^{*}(\vec{r})\right] .
\end{aligned}
$$

We suppose here that the field operators are given in the Schrödinger representation, the spinor components of the electron-positron operators being defined in the standard way [17]

$$
\begin{aligned}
& \hat{\psi}_{\nu}(\vec{r})=\sum_{s} \int \frac{d \vec{p}}{(2 \pi)^{3 / 2}}\left\{a_{\vec{p} s} u_{\vec{p} s \nu} e^{i \vec{p} \vec{r}}+b_{\vec{p} s}^{+} v_{-\vec{p}-s \nu} e^{-i \vec{p} r}\right\}, \\
& \hat{\psi}_{\nu}^{*}(\vec{r})=\sum_{s} \int \frac{d \vec{p}}{(2 \pi)^{3 / 2}}\left\{a_{\vec{p} s}^{+} u_{\vec{p} s \nu}^{*} e^{-i \vec{p} \vec{r}}+b_{\vec{p} s} v_{-\vec{p}-s \nu}^{*} e^{i \vec{p} \vec{r}}\right\} .
\end{aligned}
$$

In these formulas $\hbar=c=1$; the primary charge $\left(-e_{0}\right), e_{0}>0$ and $m_{0}$ are considered as the parameters of the model; the symbol $: \hat{H}$ : means the normal ordering of the operators excluding the vacuum energy [4]; $\vec{\alpha}, \beta$ are Dirac matrices; $u_{\vec{p} s \nu}$ and $v_{\vec{p} s \nu}$ are the components of the bispinors corresponding to the solutions of Dirac equation for the free "bare" electron and positron with the momentum $\vec{p}$ and spin $s ; a_{\vec{p} s}\left(a_{\vec{p} s}^{+}\right)$and $b_{\vec{p} s}\left(b_{\vec{p} s}^{+}\right)$are the annihilation (creation) operators for the "bare" electrons and positrons in the corresponding states. The field operator $\hat{\vec{A}}(\vec{r})$ and the operator of the photon number $\hat{n}_{\vec{k} \lambda}$ are related to the transversal electromagnetic field and their explicit form will be written below.

This Hamiltonian corresponds to the Coulomb gauge [17], when the electron-positron field interacts with the scalar field and with the transversal photons of the electromagnetic field. It will be shown below that the reconstruction of the QED gauge symmetry connected with the longitudinal field does not change the form of the considered one-particle excitation. In the Coulomb gauge the operators of the scalar field

$$
\hat{\varphi}(\vec{r})=\sqrt{4 \pi} \int d \vec{k} \hat{\varphi}_{\vec{k}} e^{i \vec{k} \vec{r}}
$$

can be excluded from the Hamiltonian [17]. For that purpose one should use the solution of the operator equations of motion for $\hat{\varphi}_{\vec{k}}$ assuming that the "bare" electrons are point-like particles and "self-action" is equivalent to the substitution of the initial mass for the renormalized one. As a result the terms with scalar fields in the Hamiltonian are reduced to the Coulomb interaction between the charged particles. However, this transformation of the Hamiltonian (2) can not be used in this paper because only the dynamics of the mass renormalization is the subject under investigation.

There is another problem connected with a negative sign of the term corresponding to the self-energy of the scalar field. If the non-relativistic problems were considered then the operator of the particle kinetic energy would be positively defined and the negative operator with the square-law dependence on $\hat{\varphi}(\vec{r})$ would lead to the "fall on the center" [18] as the energy minimum would be reached at an infinitely large field amplitude. However, if the relativistic fermion field is considered then the operator of the free particle energy (the first term in formula (2)) is not positively defined. Besides, the states of the system with the negative energy are filled. Therefore, the stable state of the system corresponds to the energy extremum(!) (the minimum one for electron and the maximum one for positron excited states). It can be reached at the 
finite value of the field amplitude (see below). The same reasons enable one to successfully use the states with indefinite metric [2] in QED although it leads to some difficulties in the non-relativistic quantum mechanics.

According to our main assumption about the large value of the initial coupling constant $e_{0}$ we are to realize the nonperturbative description of the excited state which is the nearest to the vacuum state of the system. The basic method for the nonperturbative estimation of the energy is the variational approach with some trial state vector $\left|\Phi_{1}\right\rangle$ for the approximate description of the one-particle excitation. The qualitative properties of the self-consistent excitation in the strong coupling limit [13] show that such trial vector should correspond to the general form of the wave packet formed by the one-particle excitations of the "bare" electron-positron field. Besides, the effect of polarization and the appearance of the electrostatic field $\varphi(\vec{r})$ should be taken into account, so we consider $\left|\Phi_{1}\right\rangle$ to be the eigenvector for the operator of the scalar field. Now, let us introduce the following trial vector depending on the set of variational classical functions $U_{\vec{q} s}, V_{\vec{q} s}, \varphi(\vec{r})$ for the approximate description of the quasi-particle excited state of the system:

$$
\begin{aligned}
& \left|\Phi_{1}\right\rangle \simeq\left|\Phi_{1}^{(0)}\left(U_{\vec{q} s} ; V_{\vec{q} s} ; \varphi(\vec{r})\right)\right\rangle=\int d \vec{q}\left\{U_{\vec{q} s} a_{\vec{q} s}^{+}+V_{\vec{q} s} b_{\vec{q} s}^{+}\right\}|0 ; 0 ; \varphi(\vec{r})\rangle, \\
& \hat{\varphi}(\vec{r})|0 ; 0 ; \varphi(\vec{r})\rangle=\varphi(\vec{r})|0 ; 0 ; \varphi(\vec{r})\rangle, \quad a_{\vec{q} s}|0 ; 0 ; \varphi(\vec{r})\rangle=b_{\vec{q} s}|0 ; 0 ; \varphi(\vec{r})\rangle=0 .
\end{aligned}
$$

The ground state of the system is $\left|\Phi_{0}\right\rangle=|0 ; 0 ; 0\rangle$, if we use the same notation. It corresponds to the vacuum of both interacting fields.

Firstly, let us consider the excitation with the zero total momentum. Then the constructed trial vector should satisfy the normalized conditions resulting from the definition of the total momentum $\vec{P}$ and the observed charge $e$ of the "physical" particle:

$$
\begin{aligned}
\left\langle\Phi_{1}^{(0)}|\hat{\vec{P}}| \Phi_{1}^{(0)}\right\rangle & =\sum_{s} d \vec{q} \vec{q}\left[\left|U_{\vec{q} s}\right|^{2}+\left|V_{\vec{q} s}\right|^{2}\right]=\vec{P}=0, \quad \sum_{s} d \vec{q}\left[\left|U_{q s}\right|^{2}+\left|V_{q s}\right|^{2}\right]=1, \\
\left\langle\Phi_{1}^{(0)}|\hat{Q}| \Phi_{1}^{(0)}\right\rangle & =e_{0} \sum_{s} d \vec{q}\left[\left|V_{q s}\right|^{2}-\left|U_{q s}\right|^{2}\right]=e
\end{aligned}
$$

The condition (4) requires that the functions $U_{q s}$ and $V_{q s}$ should depend on the modulus of the vector $\vec{q}$ only. Besides, one should take into account that the trial vector $\left|\Phi_{1}^{(0)}\right\rangle$ is not the accurate eigenvector of the exact integrals of motion $\hat{Q}$ and $\hat{\vec{P}}$ as it represents the accurate eigenvector of the Hamiltonian $\left|\Phi_{1}\right\rangle$ only approximately. Therefore, in the considered zero approximation the conservation laws for momentum and charge can be satisfied only on average, and this leads to the above written normalized conditions. Generally, equation (5) should not be considered as the additional condition for the variational parameters but as the definition of the observed charge of the "physical" particle at the given value of the initial charge of the "bare" particle. Therefore the sign of the observed charge is not fixed a priori. Calculating the sequential approximations to the exact state vector $\left|\Phi_{1}\right\rangle$ (see Section 4) should restore the accurate integral of motion as well. An analogous problem appears in the "polaron" theory when the momentum conservation law was taken into account for the case of the strong coupling (for example, [14, 19]).

The trial vector $\left|\Phi_{1}\right\rangle$ is actually the collective excitation of the system and in this respect the variational approach differs greatly from the perturbation theory where the zero approximation for a one-particle state is described by one of the following state vectors:

$$
\left|\Phi_{1}^{(P T)} e\right\rangle=a_{\vec{P} s}^{+}|0 ; 0 ; 0\rangle, \quad\left|\Phi_{1}\right\rangle \simeq\left|\Phi_{1}^{(P T)} p\right\rangle=b_{\vec{P} s}^{+}|0 ; 0 ; 0\rangle .
$$

These vectors do not depend on any parameters and are eigenvectors of the momentum and charge operators. But they correspond to one-particle excitations determined by the charge $e_{0}$ of 
the "bare" electron and the field $\varphi(\vec{r})=0$. We suppose that the introduction of the variational parameters into the wave function of the zero approximation will enable us to take into account the vacuum polarization.

It should be noticed that the another reason of inconsistency of the states (6) as the "physical" electron states because of the low frequency photon field was considered recently in [20].

So, the following variational estimation for the energy $E_{1}(0)=E_{1}(\vec{P}=0)$ of the state corresponding to the "physical" quasi-particle excitation of the whole system is considered in the strong coupling zero approximation:

$$
E_{1}(0) \simeq E_{1}^{(0)}\left[U_{q s} ; V_{q s} ; \varphi(\vec{r})\right]=\left\langle\Phi_{1}^{(0)}|\hat{H}| \Phi_{1}^{(0)}\right\rangle,
$$

where the average is calculated with the full Hamiltonian (2) and the functions $U_{q s}, V_{q s}, \varphi(\vec{r})$ are to be found as the solutions of variational equations

$$
\frac{\partial E_{1}^{(0)}\left(U_{q s} ; V_{q s} ; \varphi(\vec{r})\right)}{\partial U_{q s}}=\frac{\partial E_{1}^{(0)}}{\partial V_{q s}}=\frac{\partial E_{1}^{(0)}}{\partial \varphi(\vec{r})}=0
$$

with the additional conditions (4), (5).

It is quite natural, that the ground state energy is calculated in the framework of the considered approximation as follows:

$$
E_{0} \simeq E_{0}^{(0)}=\left\langle\Phi_{0}|\hat{H}| \Phi_{0}\right\rangle=0 .
$$

Further discussion is needed in connection with the application of the variational principle $(7),(8)$ for estimating the energy of the excited state, because usually the variational principle is used for estimating the ground state energy only. As far as we know it was first applied in [21] for nonperturbative calculation of the excited states of the anharmonic oscillator with an arbitrary value of anharmonicity. This approach was called the "principle of the minimal sensitivity". It was shown in our paper [22], that the application of the variational principle to the excited states is actually the consequence of the fact that the set of eigenvalues for the full Hamiltonian does not depend on the choice of the representation for eigenfunctions. As a result, the operator method for solving Schrödinger equation was developed as the regular procedure for calculating further corrections to the zero-order approximation. Later this method was applied to a number of real physical systems and proved to be very effective when calculating the energy spectrum in the wide range of the Hamiltonian parameters and quantum numbers $([23,24,25]$ and the cited references).

The average value in equation (7) is calculated neglecting the classical components of the vector field. They appear in the high-order corrections that are defined by the renormalized charge $e \ll 1$ and can be considered by means of the canonical perturbation theory (Section 5). It means that

$$
\left\langle\Phi_{1}^{(0)}\left|\hat{\psi}^{*}(\vec{r})[\vec{\alpha} \hat{\vec{A}}(\vec{r})] \hat{\psi}(\vec{r})\right| \Phi_{1}^{(0)}\right\rangle=0 .
$$

It should be noted that the possibility of constructing self-consistently the renormalized QED at the non-zero vacuum value of the scalar field operator was considered before [26] but the solution of the corresponding equations was not discussed.

Then the functional for determining the zero approximation for the energy of the one-particle excitation is defined as follows:

$$
\begin{aligned}
E_{1}(0)= & \int d \vec{r} \int \frac{d \vec{q}}{(2 \pi)^{3 / 2}} \int \frac{d \vec{q}^{\prime}}{(2 \pi)^{3 / 2}} \sum_{s, s^{\prime}} \sum_{\mu, \nu}\left\{U_{q^{\prime} s^{\prime}}^{*} u_{\vec{q}^{\prime} s^{\prime} \mu}^{*}\left[\left(\vec{\alpha} \vec{q}+\beta m_{0}\right)_{\mu \nu}+e_{0} \varphi(\vec{r}) \delta_{\mu \nu}\right] U_{q s} u_{\vec{q} s \nu}\right. \\
& \left.-V_{q^{\prime} s^{\prime}} v_{\vec{q}^{\prime} s^{\prime} \mu}^{*}\left[\left(\vec{\alpha} \vec{q}+\beta m_{0}\right)_{\mu \nu}+e_{0} \varphi(\vec{r}) \delta_{\mu \nu}\right] V_{q s}^{*} v_{\vec{q} s \nu}\right\} e^{i\left(\vec{q}-\vec{q}^{\prime}\right) \vec{r}}-\frac{1}{2} \int d \vec{r}[\vec{\nabla} \varphi(\vec{r})]^{2}
\end{aligned}
$$


In order to vary the introduced functional let us define the spinor wave functions (not operators) which describe the coordinate representation for the electron and positron wave packets in the state vector $\left|\Phi_{1}^{(0)}\right\rangle$ :

$$
\Psi_{\nu}(\vec{r})=\int \frac{d \vec{q}}{(2 \pi)^{3 / 2}} \sum_{s} U_{q s} u_{\vec{q} s \nu} e^{i \vec{q} \vec{r}}, \quad \Psi_{\nu}^{c}(\vec{r})=\int \frac{d \vec{q}}{(2 \pi)^{3 / 2}} \sum_{s} V_{q s}^{*} v_{\vec{q} s \nu} e^{i \vec{q} \vec{r}}
$$

In particular, if the trial state vector is chosen in one of the forms (6) of the standard perturbation theory, the wave functions (10) coincide with the plane wave solutions of the free Dirac equation. For a general case the variation of the functional (9) by the scalar field leads to

$$
\begin{gathered}
E(0)=\int d \vec{r}\left\{\Psi^{+}(\vec{r})\left[\left(-i \vec{\alpha} \vec{\nabla}+\beta m_{0}\right)+\frac{1}{2} e_{0} \varphi(\vec{r})\right] \Psi(\vec{r})\right. \\
-\Psi^{+c}(\vec{r})\left[\left(-i \vec{\alpha} \vec{\nabla}+\beta m_{0}\right)+\frac{1}{2} e_{0} \varphi(\vec{r})\right] \Psi^{c}(\vec{r}), \\
\int d \vec{r}\left[\Psi^{+}(\vec{r}) \Psi(\vec{r})+\Psi^{+c}\left(\vec{r}^{\prime}\right) \Psi^{c}\left(\vec{r}^{\prime}\right)\right]=1, \\
\varphi(\vec{r})=\frac{e_{0}}{4 \pi} \int \frac{d \vec{r}^{\prime}}{\left|\vec{r}-\vec{r}^{\prime}\right|}\left[\Psi^{+}\left(\vec{r}^{\prime}\right) \Psi\left(\vec{r}^{\prime}\right)-\Psi^{+c}\left(\vec{r}^{\prime}\right) \Psi^{c}\left(\vec{r}^{\prime}\right)\right] .
\end{gathered}
$$

The main condition for the existence of the considered nonperturbative excitation in QED is defined by the extremum of the functional (11) corresponding to a non-zero classical field. The structure of this functional shows that such solutions of the variational equations could appear only if the trial state vector simultaneously included the superposition of the electron and positron wave packets. So, such solutions cannot be obtained by means of the perturbation theory with the state vectors (6).

Equation (11) and the Fourier representation (3) for the trial vector clearly indicate that the assumption concerning the localization of the functions $\Psi(\vec{r})$ near some point does not contradict to the translational symmetry of the system because this point by itself can be situated at any point of the full space with equal probability. The general analysis of the correlation between the local breaking of the symmetry and the conservation of accurate integral of motion for the arbitrary quantum system was considered in detail by Bogoluibov in his widely known paper "On quasi-averages" [27]. A similar analysis of the problem in question will be given in Section 4.

Varying the functional (11) by the wave functions $\Psi(\vec{r})$ and $\Psi^{c}(\vec{r})$ taking into account their normalization conditions leads to the equivalent Dirac equations describing the electron (positron) motion in the field of potential $\varphi(\vec{r})$ :

$$
\left\{\left(-i \vec{\alpha} \vec{\nabla}+\beta m_{0}\right)+e_{0} \varphi(\vec{r})\right\} \Psi(\vec{r})=0, \quad\left\{\left(-i \vec{\alpha} \vec{\nabla}+\beta m_{0}\right)+e_{0} \varphi(\vec{r})\right\} \Psi^{c}(\vec{r})=0 .
$$

But it is important that in spite of the normalization condition (11) for the total state vector (10) each of its components could be normalized differently

$$
\int d \vec{r} \Psi^{+}(\vec{r}) \Psi(\vec{r})=\frac{1}{1+C}, \quad \int d \vec{r} \Psi^{+c}\left(\vec{r}^{\prime}\right) \Psi^{c}\left(\vec{r}^{\prime}\right)=\frac{C}{1+C} .
$$

The constant $C$ is an arbitrary value up to now. It defines the ratio of two charge states in the considered wave packet. As a result the self-consistent potential $\varphi(\vec{r})$ of the scalar field depends on $C$ because of the equation (12).

We should discuss the procedure of separating variables in more detail, because of the nonlinearity of the obtained system of equations for the wave functions and the self-consistent potential. Since the considered physical system has no preferred vectors if $\vec{P}=0$, it is natural 
to regard the self-consistent potential as spherically symmetrical. Then the variable separation for the Dirac equation is realized on the basis of the well known spherical bispinors [2]:

$$
\Psi_{j l M}=\left(\begin{array}{c}
g(r) \Omega_{j l M} \\
i f(r) \Omega_{j l^{\prime} M}
\end{array}\right)
$$

Here $\Omega_{j l M}$ are the spherical spinors [2] describing the spin and angular dependence of the one-particle excitation wave functions; $j, M$ are the total excitation momentum and its projection respectively, the orbital momentum eigenvalues are connected by the correlation $l+l^{\prime}=2 j$. It is natural to consider the state with the minimal energy as the most symmetrical one, corresponding to the values $j=1 / 2, M= \pm 1 / 2, l=0 ; l^{\prime}=1$. This choice corresponds to the condition according to which in the non-relativistic limit the "large" component of the bispinor $\Psi \sim g$ corresponds to the electronic zone of the electron-positron field. Then the unknown radial functions $f, g$ satisfy the following system of the equations:

$$
\begin{aligned}
& \frac{d(r g)}{d r}-\frac{1}{r}(r g)-\left(m_{0}-e_{0} \varphi(r)\right)(r f)=0, \\
& \frac{d(r f)}{d r}+\frac{1}{r}(r f)-\left(m_{0}+e_{0} \varphi(r)\right)(r g)=0 .
\end{aligned}
$$

The states with various projections of the total momentum should be equally populated in order to be consistent with the assumption of the potential spherical symmetry with the equation (12). So, the total wave function of the "electronic" component of the quasi-particle excitation of the electron positron field is chosen in the following form:

$$
\begin{aligned}
& \Psi=\frac{1}{\sqrt{2}}\left[\Psi_{1 / 2,0,1 / 2}+\Psi_{1 / 2,0,-1 / 2}\right]=\left(\begin{array}{c}
g(r) \chi_{0}^{+} \\
i f(r) \chi_{1}^{+}
\end{array}\right), \\
& \chi_{l}^{+}=\frac{1}{\sqrt{2}}\left[\Omega_{1 / 2, l, 1 / 2}+\Omega_{1 / 2, l,-1 / 2}\right], \quad l=0,1 .
\end{aligned}
$$

In its turn, the wave function $\Psi^{c}$ is defined on the basis of the following bispinor:

$$
\Psi_{j l M}^{c}=\left(\begin{array}{c}
-i f_{1}(r) \Omega_{j l M} \\
g_{1}(r) \Omega_{j l^{\prime} M}
\end{array}\right) \text {. }
$$

The radial wave functions $f_{1}, g_{1}$ in this case satisfy the following system of equations

$$
\begin{aligned}
& \frac{d\left(r g_{1}\right)}{d r}+\frac{1}{r}\left(r g_{1}\right)-\left(m_{0}+e_{0} \varphi(r)\right)\left(r f_{1}\right)=0, \\
& \frac{d\left(r f_{1}\right)}{d r}-\frac{1}{r}\left(r f_{1}\right)-\left(m_{0}-e_{0} \varphi(r)\right)\left(r g_{1}\right)=0 .
\end{aligned}
$$

These equations correspond to the positronic branch of the electron-positron field with the "large" component $\sim g_{1}$ in the non-relativistic limit.

It is important to note that the functions $\Psi$ and $\Psi^{c}$ satisfy the equations (13) that have the same form. It imposes an additional condition of the orthogonality on them:

$$
\left\langle\Psi^{c} \mid \Psi\right\rangle=0 .
$$

Taking into account this condition and also the requirement that the states with different values of $M$ should be equally populated one finds the "positronic" wave function

$$
\begin{aligned}
& \Psi^{c}=\frac{1}{\sqrt{2}}\left[\Psi_{1 / 2,0,1 / 2}^{c}-\Psi_{1 / 2,0,-1 / 2}^{c}\right]=\left(\begin{array}{c}
-i f_{1}(r) \chi_{0}^{-} \\
g_{1}(r) \chi_{1}^{-}
\end{array}\right), \\
& \chi_{l}^{-}=\frac{1}{\sqrt{2}}\left[\Omega_{1 / 2, l, 1 / 2}-\Omega_{1 / 2, l,-1 / 2}\right], \quad l=0,1 .
\end{aligned}
$$


The equation for the self-consistent potential follows from the definition of $\varphi(r)$ in formula (12) taking into account the normalization of the spherical spinors [2]:

$$
\frac{d^{2} \varphi}{d r^{2}}+\frac{2}{r} \frac{d \varphi}{d r}=-\frac{e_{0}}{4 \pi}\left[f^{2}+g^{2}-f_{1}^{2}-g_{1}^{2}\right]
$$

The boundary condition for the potential is equivalent to the normalization condition (5) and defines the charge $e$ of the "physical" electron (positron)

$$
\left.\varphi(r)\right|_{r \rightarrow \infty}=\frac{e}{4 \pi r}=\frac{e_{0}}{4 \pi r} \int_{0}^{\infty} r_{1}^{2} d r_{1}\left[f^{2}\left(r_{1}\right)+g^{2}\left(r_{1}\right)-f_{1}^{2}\left(r_{1}\right)-g_{1}^{2}\left(r_{1}\right)\right] .
$$

It is important to stress that the form of the functions given above is defined practically uniquely by the imposed conditions. At the same time the obtained equations are consistent with the symmetries defined by the physical properties of the system. The first symmetry is quite evident and relates to the fact that the excitation energy does not depend on the choice of the quantization axis of the total angular momentum.

Moreover, these equations satisfy the condition of the charge symmetry [2]. Indeed, by direct substitution, one can check that one more pair of bispinors leads to the equations completely coinciding with (15), (18)

$$
\begin{aligned}
\tilde{\Psi}_{j l M} & =\left(\begin{array}{c}
i g_{1}(r) \Omega_{j l^{\prime} M} \\
-f_{1}(r) \Omega_{j l M}
\end{array}\right), \\
\tilde{\Psi}_{j l M}^{c} & =\left(\begin{array}{c}
-f(r) \Omega_{j l^{\prime} M} \\
-i g(r) \Omega_{j l M}
\end{array}\right) .
\end{aligned}
$$

It means that these bispinors allow one to find another pair of the wave functions which are orthogonal to each other and to the functions (16), (20) but include the same set of the radial functions

$$
\tilde{\Psi}=\left(\begin{array}{c}
i g_{1}(r) \chi_{1}^{-} \\
-f_{1}(r) \chi_{0}^{-}
\end{array}\right), \quad \tilde{\Psi}^{c}=\left(\begin{array}{c}
-f(r) \chi_{1}^{+} \\
-i g(r) \chi_{0}^{+}
\end{array}\right) .
$$

These functions differ from the set (16), (20) because they lead to a different sign of the observed charge of the quasi-particle due to the boundary condition (22) and describe the "physical" positron.

The structure of the equation (13) shows that the considered variational method is consistent with the gauge symmetry of the initial Hamiltonian. One can see that these equations are invariant with respect to the following transformations:

$$
\begin{aligned}
& \vec{\nabla} \Rightarrow \vec{\nabla}+i e_{0} \vec{A}_{l}(\vec{r}), \\
& \Psi(\vec{r}) \Rightarrow e^{-i \beta(\vec{r})} \Psi(\vec{r}), \quad \vec{\nabla} \beta(\vec{r})=e_{0} \vec{A}_{l}(\vec{r}),
\end{aligned}
$$

with an arbitrary longitudinal potential $\overrightarrow{A_{l}}(\vec{r})$.

It means that the Hamiltonian (2) could be chosen in an arbitrary Lorentz gauge with the classical components both for the scalar field $\varphi(\vec{r})$ and for the longitudinal field $\overrightarrow{A_{l}}(\vec{r})$ if the following condition was fulfilled:

$$
\Delta \beta(\vec{r})=\rho_{l}(\vec{r})=0, \quad \rho_{l}(\vec{r})=\Psi^{+}(\vec{r})(\vec{r} \cdot \vec{\nabla}) \Psi(\vec{r}) .
$$

One can easily check that the condition $\rho_{l}(\vec{r})=0$ is fulfilled identically for the functions that describes the quasi-particle above.

Let us now proceed to the solution of the variational equations. It follows from the qualitative analysis that the important property of the trial state vector is the possibility to vary the relative 
contribution of the electronic and positronic components of the wave function. Therefore let us introduce the variational parameter $C$ in the following way:

$$
\int_{0}^{\infty} r^{2} d r\left[f^{2}(r)+g^{2}(r)\right]=\frac{1}{1+C}, \quad \int_{0}^{\infty} r^{2} d r\left[f_{1}^{2}(r)+g_{1}^{2}(r)\right]=\frac{C}{1+C} .
$$

The dimensionless variables and new functions can be introduced

$$
\begin{aligned}
& x=r m_{0}, \quad E=\epsilon m_{0}, \quad e_{0} \varphi(r)=m_{0} \phi(x), \quad \frac{e_{0}^{2}}{4 \pi}=\alpha_{0}, \quad u(x) \sqrt{m_{0}}=r g(r), \\
& v(x) \sqrt{m_{0}}=r f(r), \quad u_{1}(x) \sqrt{m_{0}}=r g_{1}(r), \quad v_{1}(x) \sqrt{m_{0}}=r f_{1}(r) .
\end{aligned}
$$

As a result the system of equations for describing the radial wave functions of the oneparticle excitation of the electron-positron field and the self-consistent potential of the vacuum polarization can be obtained:

$$
\begin{aligned}
& \frac{d u}{d x}-\frac{1}{x} u-(1-\phi(x)) v=0, \quad \frac{d v}{d x}+\frac{1}{x} v-(1+\phi(x)) u=0, \\
& \frac{d u_{1}}{d x}+\frac{1}{x} u_{1}-(1+\phi(x)) v_{1}=0, \quad \frac{d v_{1}}{d x}-\frac{1}{x} v_{1}-(1-\phi(x)) u_{1}=0, \\
& \phi(x)=\alpha_{0}\left[\int_{x}^{\infty} d y \frac{\rho(y)}{y}+\frac{1}{x} \int_{0}^{x} d y \rho(y)\right], \quad \rho(x)=\left[u^{2}(x)+v^{2}(x)-u_{1}^{2}(x)-v_{1}^{2}(x)\right] .
\end{aligned}
$$

The mathematical structure of equations (27) is analogous to that of the self-consistent equations for localized state of "polaron" in the strong coupling limit [14]. Therefore the same approach can be used for the numerical solution of these nonlinear equations. It has been developed and applied [28] for the "polaron" problem on the basis of the continuous analog of Newton's method.

Let us take into account that the system of equations (27) can be simplified because the pairs of the functions $u, v$ and $u_{1}, v_{1}$ are satisfied by the same equations and differ only by the normalized condition. Therefore they can be represented by means of one pair of functions if special notations are used:

$$
\begin{aligned}
& u=\sqrt{\frac{1}{1+C}} u_{0}, \quad v=\sqrt{\frac{1}{1+C}} v_{0}, \quad u_{1}=\sqrt{\frac{C}{1+C}} v_{0}, \quad v_{1}=\sqrt{\frac{C}{1+C}} u_{0}, \\
& \int_{0}^{\infty} d x\left[u_{0}^{2}(x)+v_{0}^{2}(x)\right]=1, \quad \rho_{0}(x)=u_{0}^{2}(x)+v_{0}^{2}(x), \\
& \frac{d u_{0}}{d x}-\frac{1}{x} u_{0}-(1-\phi(x)) v_{0}=0, \quad \frac{d v_{0}}{d x}+\frac{1}{x} v_{0}-(1+\phi(x)) u_{0}=0, \\
& \phi(x)=\alpha_{0} \frac{1-C}{1+C} \phi_{0}(x), \quad \phi_{0}(x)=\int_{x}^{\infty} d y \frac{\rho_{0}(y)}{y}+\frac{1}{x} \int_{0}^{x} d y \rho_{0}(y) .
\end{aligned}
$$

The energy of the system (11) can also be calculated by these functions:

$$
\begin{aligned}
& E_{1}(0) \equiv E(0)=m_{0} \frac{1-C}{1+C}\left[T+\frac{1}{2} \alpha_{0} \frac{1-C}{1+C} \Pi\right], \\
& T=\int_{0}^{\infty} d x\left[\left(u_{0}^{\prime} v_{0}-v_{0}^{\prime} u_{0}\right)-2 \frac{u_{0} v_{0}}{x}+\left(u_{0}^{2}-v_{0}^{2}\right)\right], \quad \Pi=\int_{0}^{\infty} d x \phi_{0}\left(u_{0}^{2}+v_{0}^{2}\right) .
\end{aligned}
$$

and equation (28) can be obtained when varying of the functional (29).

The required solutions are to be normalized and this condition defines the asymptotic behavior of the functions near the integration interval boundaries:

$$
\begin{aligned}
& u_{0} \approx A x\left[1+\frac{1-\phi^{2}(0)}{6} x^{2}\right], \quad v_{0} \approx A \frac{1-\phi(0)}{3} x^{2}, \quad x \rightarrow 0, \\
& u_{0} \approx A_{1} \exp (-x), \quad v_{0} \approx-A_{1} \exp (-x), \quad x \rightarrow \infty .
\end{aligned}
$$




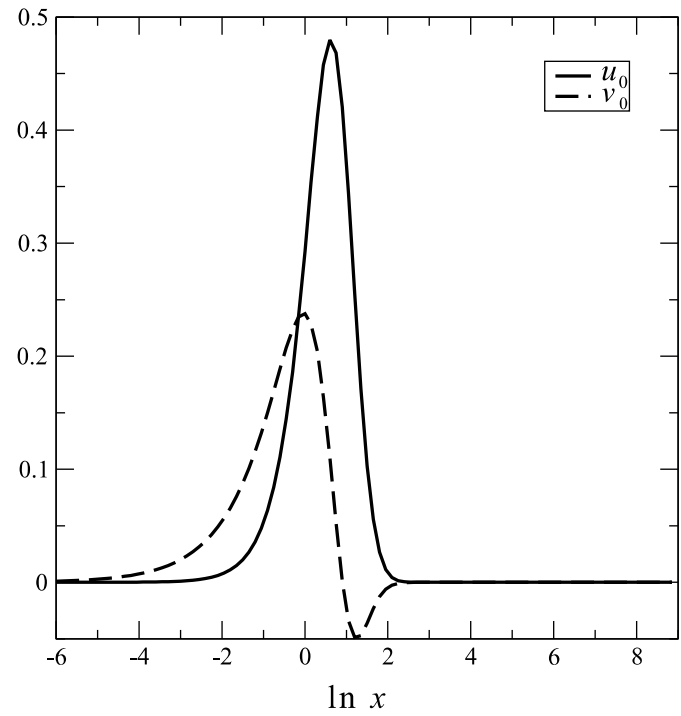

Figure 1. Localized wave functions of the quasiparticle excitation; $x=r / r_{0}=\frac{2\left|a_{0}\right| r}{r_{e}}, u_{0}=$ $\xi x g\left(x r_{0}\right), v_{0}=u_{0}=\xi x f\left(x r_{0}\right), \xi=\frac{1}{4}\left(\frac{\alpha}{\left|a_{0}\right| m}\right)^{3 / 2}$.

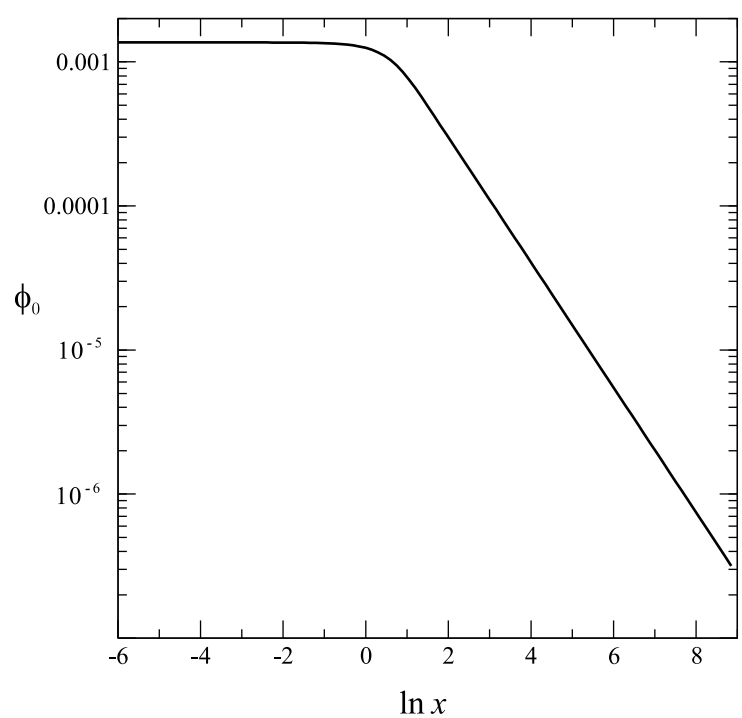

Figure 2. Self-consistent potential of the excitation; $x=r / r_{0}=\frac{2\left|a_{0}\right| r}{r_{e}}, \phi_{0}(x)=\frac{e}{2\left|a_{0}\right| m} \varphi\left(x r_{0}\right)$.

The value

$$
a=\alpha_{0} \frac{1-C}{1+C}
$$

is the free parameter of the equations (28) and it plays a role of the eigenvalue when the nontrivial normalized solution exists.

The method for the numerical solution of the nonlinear self-consistent system of the equations (28) was described in detail in the paper [29]. Only the numerical results for the localized wave functions and for the scalar potential are described in the present work. Certainly, the numerical value for the parameter $a$ depends on the accuracy of the finite-difference approximation for the differential operators in the whole interval of integration. The value $a$ was calculated more accurately in comparison with [29]:

$$
a=a_{0} \approx-3.531 .
$$

Fig. 1 shows the solutions $u_{0}, v_{0}$ for the electron and positron components of the excitation that are localized in the domain with the linear size of $\sim m_{0}^{-1}$. Fig. 2 represents the selfconsistent potential $\phi_{0}$ that provides stability of the system in this domain and corresponds to the value $a_{0}$. It gets over the Coulomb potential of the "physical" charge $e$ for $r>r_{0}=m_{0}^{-1}$. It is important that the characteristic size of this excitation $r_{0}$ is the same order as the classical radius of the electron $r_{e}=\alpha / m$, namely $r_{0}=\frac{r_{e}}{2\left|a_{0}\right|} \approx 0.15 r_{e}$ (see below equation (38)).

\section{Physical interpretation of the quasi-particle excitation and estimation of the $\mu$-meson mass}

The stationary localized collective excitation of the electron-positron field described above is of great interest by itself as the eigenvector of the well known QED Hamiltonian that cannot be calculated by means of the perturbation theory and has not be considered before. But it is also essential to find its physical interpretation because the only stable objects observed in 
the electrodynamic processes are electrons (positrons) and photons. Therefore it is natural to suppose that this localized state describes the "physical" electron (positron) with the observed charge $e$. The integral charge of the considered one-particle excitation is defined by the boundary condition (22) and this supposition leads to:

$$
e_{0} \frac{(1-C)}{(1+C)}=e \text {. }
$$

Taking into account the definition (30) one can found the following relation between the "primary" coupling constant $\alpha_{0}=e_{0}^{2} / 4 \pi$ and the observed value of the fine structure constant $\alpha=e^{2} / 4 \pi$

$$
\alpha_{0}=\frac{a_{0}^{2}}{\alpha} \approx 1708.1 \text {. }
$$

This formula defines the renormalization of the charge in the considered approximation and shows self-consistency of the initial supposition that the interaction between the "primary" electron-positron and scalar fields is strong. Then the renormalization constant [2] $\left(\alpha=Z(\alpha) \alpha_{0}\right)$ is:

$$
Z^{(0)}(\alpha)=\frac{\alpha^{2}}{a_{0}^{2}}
$$

It should be stressed once more that the large value of the "primary" coupling constant $\alpha_{0}$ does not mean at all that the perturbation theory cannot be applied for the calculation of the observed physical values. In connection with it let us remind that the "primary" coupling constant in the existed form of QED tends to infinity $\left(\alpha_{0} \rightarrow \infty\right)$ because of "Landau pole" [6, $\S 128]$. Nevertheless, it can be excluded from the observed values by means of the renormalization procedure. In our representation the large but finite value of $\alpha_{0}$ is important only for the formation of the initial basis of the self-localized states but it is also excluded when calculating the observed physical values in a power series of the "physical" coupling constant $\alpha \simeq \alpha_{0}^{-1} \ll 1$. However, in this case one can avoid the divergent integrals when performing the renormalization procedure (see below Section 5).

The QED Hamiltonian (2) is defined with $e_{0}>0$ and the integral charge of the quasiparticle $e<0$ because of the conditions (30) and (31). It means that the considered excitation corresponds to the "physical" electron. The excitation corresponding to the "physical" positron is defined by the bispinors (23).

The change of the integral charge of the excitation is explained by the local intersection of the electron and positron energy zones in the strong self-consistent scalar electromagnetic field. The analogous states appear also in the strong Coulomb field of the nucleus with the large charge $Z>137$ when the gap between Dirac zones tends to zero and stabilization of the electron states is achieved due to creation of the additional positrons (see, for example, [30] and references therein). In our case the origin of the charge density of one sign is the source of the strong scalar electromagnetic field that leads to coming together the electron and positron levels. This field can be compensated by the extra value of the charge density of opposite sign. More detailed qualitative analysis of the structure of the quasi-particle excitation was discussed in the paper [31]. It is also interesting to note that the relation between the charges of the "bare" and "physical" electrons has the same form as the equation for the electric and magnetic charges in the theory of the Dirac monopole [32].

The eigenvalue $a_{0}$ corresponds to minimum of the functional (29) with fixed functions $u_{0}$, $v_{0}, \varphi_{0}$ with respect to the parameter $\xi=(1-C) /(1+C)$ and can be expressed in terms of the integrals $T$ and $\Pi$ that define kinetic and potential contributions in the total excitation energy

$$
a_{0}=-\frac{T}{\Pi}
$$


and the numerical value of the integral $T$ can be calculated with the considered accuracy as

$$
T \approx 0.749 \text {. }
$$

Then the total energy of the excitation with zero momentum is:

$$
E(0)=-\frac{m_{0}}{\alpha_{0}} \frac{T a_{0}}{2}=-m_{0} \alpha \frac{T}{2 a_{0}}>0 .
$$

This value defines the minimal energy of the one-particle excitation of the electron-positron field and its positive sign corresponds to the "bottom" of the "physical" electron zone in the renormalized QED. It is also consistent with the negative charge of this excitation [31]. It is natural to consider this value as the rest-mass $m$ of the "physical" electron and to find the mass renormalization in QED:

$$
E(0)=m \equiv m_{e}=-m_{0} \alpha \frac{T}{2 a_{0}}, \quad m_{0}=m_{e} \frac{2\left|a_{0}\right|}{\alpha} \approx 1291.7 m_{e} .
$$

This relation $\left(E(0) \ll m_{0}\right)$ shows that the primary mass of the "bare" electrons and positron $\sim m_{0}$ is compensated by the binding energy of their charge distributions almost completely. It is interesting that the characteristic size of the domain, where the considered one-particle excitation is localized $\left(\Delta r \approx m_{0}^{-1}\right)$, is the same order as the value of the electron "radius" $\left(r_{e}=\alpha / m\right)$ in the classical model of Abraham-Lorentz [8].

In accordance with the formulas (33), (38) characteristics of the charge spatial distribution in the "physical" electron depend on the observable QED parameters $\alpha$ and $m$ only. Such internal structure of the electron does not contradict to the well known results (for example, [33]) that maintain that the observed characteristics of the electron cannot depend on any dimensional parameter with the exception of $m$ (see below the discussion in Section 5). Actually the local charge distribution in QED arises as well in the framework of the perturbation theory when considering the physical interpretation of the renormalization of charge (for example, [2]).

As it was shown by Dirac [8], investigation of the "physical" electron with the distributed charge is of great interest because it gives the possibility to interpret the "physical" $\mu$-meson as the excited state of such system. In order to describe the dynamics of such excitation Dirac introduced the hypothetical elastic parameter that is absent in QED. However, the variational approach considered in the present paper allows one to analyze the one-particle excitation differed from the "physical" electron without inclusion of any additional parameters.

Let us choose the trial state vector in the same form as it is given by formulas (3), (7), but with the wave functions that are orthogonal to the "physical" electron state vector. These functions are satisfied to the system of the self-consistent equations in the form (11)-(14), but with the nonzero eigenvalues $E_{1} \neq E_{2}$ for both components of the "primary" electron-positron field:

$$
\begin{aligned}
& \left\{\left(-i \vec{\alpha} \vec{\nabla}+\beta m_{0}\right)+e_{0} \varphi_{\mu}(\vec{r})-E_{1}\right\} \Psi_{\mu}(\vec{r})=0, \\
& \left\{\left(-i \vec{\alpha} \vec{\nabla}+\beta m_{0}\right)+e_{0} \varphi_{\mu}(\vec{r})-E_{2}\right\} \Psi_{\mu}^{c}(\vec{r})=0, \\
& \varphi_{\mu}(\vec{r})=\frac{e_{0}}{4 \pi} \int \frac{d \vec{r}^{\prime}}{\left|\vec{r}-\vec{r}^{\prime}\right|}\left[\Psi_{\mu}^{+}\left(\vec{r}^{\prime}\right) \Psi_{\mu}\left(\vec{r}^{\prime}\right)-\Psi_{\mu}^{+c}\left(\vec{r}^{\prime}\right) \Psi_{\mu}^{c}\left(\vec{r}^{\prime}\right)\right], \\
& \int d \vec{r}\left[\Psi_{\mu}^{+}(\vec{r}) \Psi_{\mu}(\vec{r})+\Psi_{\mu}^{+c}\left(\vec{r}^{\prime}\right) \Psi_{\mu}^{c}\left(\vec{r}^{\prime}\right)\right]=1, \quad \int d \vec{r}\left[\Psi_{\mu}^{+}(\vec{r}) \Psi(\vec{r})+\Psi_{\mu}^{+c}\left(\vec{r}^{\prime}\right) \Psi^{c}\left(\vec{r}^{\prime}\right)\right]=0, \\
& \int d \vec{r} \Psi_{\mu}^{+}(\vec{r}) \Psi_{\mu}(\vec{r})=\frac{1}{1+C}, \quad \int d \vec{r} \Psi_{\mu}^{+c}\left(\vec{r}^{\prime}\right) \Psi_{\mu}^{c}\left(\vec{r}^{\prime}\right)=\frac{C}{1+C} .
\end{aligned}
$$


In this case the energy that defines the observed mass of the "physical" $\mu$-meson can be calculated as

$$
E_{\mu}(\vec{P}=0)=m_{\mu}=E_{1}-E_{2} .
$$

The parameters $C, e_{0}, m_{0}$ in equations (38) should be the same as was defined by equations (32)-(38) because the observed charges of the "physical" electron and $\mu$-meson coincide. Besides, the same bispinors (16)-(24) as for electron should be used when separating variables in equation (39). However, the radial functions for both components of the "primary" electronpositron field that form the "physical" $\mu$-meson should correspond to the different eigenvalues.

If the dimensionless variables (26) have been used the following system of equations is obtained:

$$
\begin{array}{ll}
\frac{d u_{\mu}}{d x}-\frac{1}{x} u_{\mu}-\left(1+\epsilon_{1}-\phi_{\mu}(x)\right) v_{\mu}=0, & \frac{d v_{\mu}}{d x}+\frac{1}{x} v_{\mu}-\left(1-\epsilon_{1}+\phi_{\mu}(x)\right) u_{\mu}=0, \\
\frac{d u_{1 \mu}}{d x}+\frac{1}{x} u_{1 \mu}-\left(1-\epsilon_{2}+\phi_{\mu}(x)\right) v_{1 \mu}=0, & \frac{d v_{1 \mu}}{d x}-\frac{1}{x} v_{1 \mu}-\left(1+\epsilon_{2}-\phi_{\mu}(x)\right) u_{1 \mu}=0,(40) \\
\phi_{\mu}(x)=\alpha_{0}\left[\int_{x}^{\infty} d y \frac{\rho_{\mu}(y)}{y}+\frac{1}{x} \int_{0}^{x} d y \rho_{\mu}(y)\right], & \rho(x)=\left[u_{\mu}^{2}(x)+v_{\mu}^{2}(x)-u_{1 \mu}^{2}(x)-v_{1 \mu}^{2}(x)\right] .
\end{array}
$$

If the solution of the nonlinear problem (40) with the eigenvalues $\epsilon_{1,2}$ and the orthogonality and normalization conditions is found, the observed mass of $\mu$-meson is defined by the following formula:

$$
\begin{aligned}
& m_{\mu}=m_{0}\left(I_{\mu}-I_{1 \mu}\right), \\
& I_{\mu}=\int_{0}^{\infty} d x\left\{u_{\mu}^{\prime} v_{\mu}-u_{\mu}^{\prime} v_{\mu}-2 \frac{u_{\mu} v_{\mu}}{x}+\left(u_{\mu}^{2}-v_{\mu}^{2}\right)+\frac{1}{2} a_{0} \phi_{\mu}\left(u_{\mu}^{2}+v_{\mu}^{2}\right)\right\},
\end{aligned}
$$

where integral $I_{1 \mu}$ is expressed by the functions $u_{1 \mu}, v_{1 \mu}$ with the same formula as the integral $I_{\mu}$ by the functions $u_{\mu}, v_{\mu}$; the parameter $a_{0}$ is defined by equation (35).

Equation (37) for the observed mass of the electron can be written in the same form

$$
m_{e}=m_{0} \frac{1-C}{1+C} I_{0}
$$

where integral $I_{0}$ is expressed by the functions $u_{0}, v_{0}$ from equations $(27)$.

Calculation of the excited states for the nonlinear equations (40) with the self-consistent potential proved to be a quite difficult numerical problem. In the present paper this solution was calculated on the basis of the "adiabatic" approximation for the potential. It means that the potential was fixed in the same form as in equations (27): $\phi_{\mu} \approx \phi_{0}$. In this case the functions $u_{\mu}, v_{\mu}$ coincide with $u_{0}, v_{0}$ but the functions $u_{1 \mu}, v_{1 \mu}$ correspond to the first excited state in the Dirac equations (27). Then the relation (32) and the definitions of the integrals $I$ lead to the following formulas:

$$
\frac{1-C}{1+C}=\frac{e}{e_{0}} \ll 1, \quad I_{0} \approx I_{\mu},
$$

and the observed electron- $\mu$-meson mass ratio can be calculated as:

$$
\frac{m_{\mu}}{m_{e}} \approx \frac{1-C}{1+C} \frac{I_{\mu}-I_{1 \mu}}{2 I_{\mu}}=\frac{\left|a_{0}\right|}{2 \alpha} \frac{I_{\mu}-I_{1 \mu}}{I_{\mu}} \approx 242.2 \frac{I_{\mu}-I_{1 \mu}}{I_{\mu}} .
$$

The calculated values for the integrals are the following $I_{\mu} \approx 0.95, I_{1 \mu} \approx 0.25$ and the mass ratio is estimated as

$$
\frac{m_{\mu}}{m_{e}} \approx 194
$$


This number can be compared with the experimental value $\left(m_{\mu} / m_{e}\right)_{E} \approx 206$ and the result obtained by Dirac [8]: $\left(m_{\mu} / m_{e}\right)_{D} \approx 54$. Thus, the physical interpretation of the QED oneparticle excitations leads to a quite good estimation for the observed mass of $\mu$-meson. It can be improved if the completely self-consistent solution is found for equations (40).

It may seem that interpretation of the "physical" $\mu$-meson as the excited state of the "bare" electron-positron and electromagnetic fields contradicts to the experimentally observed conservation of the muon (not electrical) charge in the electromagnetic processes. However, let us remind that this state is the collective(!) excitation of the whole system with the scalar electromagnetic field $\varphi_{\mu}$ not equal to the field $\varphi$ for the "physical" electron self-localized state. In accordance with the formula (3) it means that the both states are defined by the coherent states corresponding to different field amplitudes. Therefore the transition between these states is defined by the overlapping integral between the corresponding coherent states of the electromagnetic field. If one uses the standard definition of such states [2] this integral can be represented in the following form

$$
I=\exp \left[-4 \pi \int \frac{d \vec{k}}{\omega_{k}}\left|\vec{E}(\vec{k})-\vec{E}_{\mu}(\vec{k})\right|^{2}\right] .
$$

Here $\vec{E}(\vec{k}), \vec{E}_{\mu}(\vec{k})$ are the Fourier components of the corresponding electric field strengths, for example:

$$
\vec{E}(\vec{k})=-\frac{1}{(2 \pi)^{3}} \int d \vec{r} \vec{\nabla} \varphi(\vec{r}) e^{i \vec{k} \cdot \vec{r}}
$$

With utilisation of the dimensionless variables (26) the integral (41) can be written as

$$
\begin{aligned}
& I=e^{-\alpha_{0} \Lambda}, \quad \Lambda=\int_{0}^{\infty} t d t\left[\Phi(t)-\Phi_{\mu}(t)\right]^{2} \\
& \Phi(t)=\int_{0}^{\infty} x d x \phi^{\prime}(x) \sin (t x), \quad \Phi_{\mu}(t)=\int_{0}^{\infty} x d x \phi_{\mu}^{\prime}(x) \sin (t x) .
\end{aligned}
$$

Parameter $\Lambda \sim 1$ is defined by the converged integrals because of the asymptotic behavior of both functions $\phi(x), \phi_{\mu}(x)$ when $x \rightarrow \infty$ and $x \rightarrow 0$. The overlapping integral (42) permits one to estimate the electromagnetic lifetime of $\mu$-meson as

$$
\tau_{\mu}^{e l} \sim m_{0}^{-1} I^{-1} \sim 10^{300}[\mathrm{sec}] .
$$

This value ensures the conservation of the muon charge in the electromagnetic processes because it is essentially bigger than the total $\mu$-meson lifetime conditioned by the weak interaction.

\section{Lorentz invariance of the self-localized state with nonzero momentum}

In the previous sections the resting quasi-particle with a non-trivial self-consistent charge distribution, the finite energy $E(0)$ and a zero total momentum $\vec{P}=0$ was considered in the framework of a nonperturbative QED.

The obtained solution allows one to imagine the internal structure of the resting "physical" electron (positron) as a strongly coupled state of charge distributions of the opposite sign. The large values of integral charges of these distributions compensate each other almost completely and their heavy masses are "absorbed" by the binding energy. Actually the energy $\pm E(0)$ defines the boundaries of the renormalized electron and positron zones resulting from the strong 
polarization of the electron-positron field when the excitation appears. But this excitation could be interpreted as the "physical" electron (positron) if the sequence of the levels in every zone determined by the vector $\vec{P} \neq 0$ were described by the Lorentz invariant relativistic energy spectrum $\pm E(\vec{P})$ of real particles, that is

$$
E(\vec{P})=\sqrt{P^{2}+E^{2}(0)}=\sqrt{P^{2}+m^{2}} .
$$

It is worth saying, that the problem of studying of the dynamics of the self-localized excitation should be solved for any system with a strong interaction between quantum fields in order to calculate its effective mass. For example, a similar problem for Pekar "polaron" [13] in the ionic crystal was considered in $[14,19,34,35]$ and in a lot of more recent works. It is essential that because of the non-linear coupling between the particle and a self-consistent field the energy dispersion $E(\vec{P})$ for the quasi-particle proves to be very complicated. As the result, its dynamics in the crystal is similar to the motion of the point "physical" particle only at a small enough total momentum.

However, in the case of QED the problem is formulated in a fundamentally different way. There is currently no doubt that the dynamics of the "physical" excitation should be described by the formula (43) for any(!) values of the momentum $\vec{P}$ because of the Lorentz invariance of the Hamiltonian. It means that the considered nonperturbative approach for describing the internal structure of the "physical" electron should lead to the energy dispersion law (43) for the entire range of the momentum $\vec{P}$.

The rigorous method of taking into account the translational symmetry in the strong coupling theory for the "polaron" problem was elaborated in the works of Bogoliubov [14] and Gross [19]. Let us remind that this method was based on the introduction of the collective variable $\vec{R}$ conjugated to the total momentum operator $\hat{\vec{P}}$. The canonical character of the transformation caused by three new variables $R_{i}$ was provided by the same number of additional conditions imposed on the other variables of the system. In the "polaron" problem the quantum field interacting with the particle contributes to the total momentum of the system. It allows one to impose these conditions on the canonical field variables [14, 19] and the concrete form of the variable transformation is based mainly on the permutation relations for the boson field operators.

The considered problem has some specific features in comparison with the "polaron" problem. Firstly, the formation of the one-particle wave packet is the multi-particle effect because this packet includes all initial states of electron-positron field as the fermion field. Secondly, its selflocalization is provided by the polarization potential of the scalar field that does not contribute to the total momentum of the system. Therefore, we use a different approach in order to select the collective coordinate $\vec{R}$. Let us return to the configuration representation in the Hamiltonian (2), where QED is considered to be the totality of $N(N \rightarrow \infty)$ point electrons interacting with the quantum electromagnetic field in the Coulomb gauge [17]:

$$
\begin{aligned}
& \hat{H}=\sum_{a=1}^{N}\left\{\vec{\alpha}_{a}\left[\hat{\vec{p}}_{a}+e_{0} \hat{\vec{A}}\left(\vec{r}_{a}\right)\right]+\beta_{a} m_{0}+e_{0} \hat{\varphi}\left(\vec{r}_{a}\right)\right\}-\frac{1}{2} \int d \vec{r}(\vec{\nabla} \hat{\varphi}(\vec{r}))^{2}+\sum_{\vec{k} \lambda} \omega(\vec{k}) \hat{n}_{\vec{k} \lambda}, \\
& \omega(\vec{k})=k, \quad \hat{n}_{\vec{k} \lambda}=c_{\vec{k} \lambda}^{+} c_{\vec{k} \lambda}, \quad \lambda=1,2, \\
& \hat{\vec{A}}(\vec{r})=\sum_{\vec{k} \lambda} \frac{1}{\sqrt{2 k \Omega}} \vec{e}^{(\lambda)}\left[c_{\vec{k} \lambda} e^{i \vec{k} \vec{r}}+c_{\vec{k} \lambda}^{+} e^{-i \vec{k} \vec{r}}\right] .
\end{aligned}
$$

Here $\Omega$ is the normalized volume; $c_{\vec{k} \lambda}^{+}\left(c_{\vec{k} \lambda}\right)$ are the operators of the creation (annihilation) of quanta of a transversal electromagnetic field, the quantum having the wave vector $\vec{k}$, polarization $\vec{e}^{(\lambda)}$ and energy $\omega(\vec{k})=k$. The sign of the interaction operators differs from the standard one because the parameter $e_{0}$ is introduced as a positive quantity. 
As it was stated above the zero approximation of nonperturbative QED is defined only by a strong interaction of electrons with the scalar field, where the interaction with the transversal field is to be taken into account later in the framework of the standard perturbation theory. So, the conservation of the total momentum in the processes with transversal electromagnetic field will be provided automatically [2]. Therefore, while describing the quasi-particle excitation we should consider the conservation of the total momentum only for the system of electrons. In the considered representation it is defined by the sum of the momentum operators of individual particles

$$
\hat{\vec{P}}=\sum_{a=1}^{N} \hat{\vec{p}}_{a}, \quad \hat{\vec{P}}\left|\Phi_{1}(\vec{P})\right\rangle=\vec{P}\left|\Phi_{1}(\vec{P})\right\rangle .
$$

It means that in the configuration space the variable $\vec{R}$ conjugated to the total momentum is simply a coordinate of the center of mass of the electron-positron system and the desired transformation to new variables is as follows:

$$
\begin{array}{ll}
\vec{r}_{a}=\vec{R}+\vec{\rho}_{a}, & \vec{R}=\frac{1}{N} \sum_{a=1}^{N} \vec{r}_{a}, \quad \sum_{a=1}^{N} \vec{\rho}_{a}=0, \quad \vec{p}_{a}=-i \vec{\nabla}_{a}=-\frac{i}{N} \vec{\nabla}_{R}+\vec{p}_{a}^{\prime}, \\
\hat{\vec{P}}=-i \vec{\nabla}_{R}, \quad \vec{p}_{a}^{\prime}=-i \vec{\nabla}_{\rho_{a}}+\frac{i}{N} \sum_{b=1}^{N} \vec{\nabla}_{\rho_{b}}, \quad \sum_{a=1}^{N} \vec{p}_{a}^{\prime}=0 .
\end{array}
$$

The Hamiltonian (44) with new variables has of the following form

$$
\begin{aligned}
\hat{H}= & \sum_{a=1}^{N}\left\{\vec{\alpha}_{a}\left[-\frac{i}{N} \vec{\nabla}_{R}+\hat{\vec{p}}_{a}^{\prime}+e_{0} \hat{\vec{A}}\left(\vec{\rho}_{a}+\vec{R}\right)\right]+\beta_{a} m_{0}+e_{0} \hat{\varphi}\left(\vec{\rho}_{a}\right)\right\} \\
& -\frac{1}{2} \int d \vec{r}(\vec{\nabla} \hat{\varphi}(\vec{r}))^{2}+\sum_{\vec{k} \lambda} \omega(\vec{k}) \hat{n}_{\vec{k} \lambda} .
\end{aligned}
$$

It should be noted that the matrix elements of an arbitrary operator in a new configuration representation are to be calculated by integration over the coordinates both of the center of mass and the relative variables. Let us introduce a special notation for this norm:

$$
\left\langle\left\langle\Phi_{1}|\hat{M}| \Phi_{2}\right\rangle\right\rangle=\int d \vec{R} \prod_{a} d \vec{\rho}_{a} \Phi_{1}^{*}\left(\vec{R},\left\{\vec{\rho}_{a}\right\}\right) \hat{M} \Phi_{2}\left(\vec{R},\left\{\vec{\rho}_{a}\right\}\right) .
$$

The interaction between the "physical" electron and the transversal electromagnetic field will be taken into account by means of the perturbation theory (see below Section 5) and only the scalar field is considered in the zeroth approximation. Let us denote by $\hat{H}_{0}$ that part of the operator (45) which does not depend on the transversal electromagnetic field and describes the internal structure of the "physical" particles in the zero approximation. In fact, the operator $\hat{H}_{0}$ does not depend on the coordinate $\vec{R}$ because of its commutativity with the operator of the total momentum of the system of electrons. This also follows from the well known result [17] that in the Coulomb gauge the scalar potential could be excluded from the Hamiltonian. As a result the operator $\hat{H}_{0}$ depends only on the vector differences $\left(\vec{r}_{a}-\vec{r}_{b}\right)=\left(\vec{\rho}_{a}-\vec{\rho}_{b}\right)$ and does not change with the simultaneous translation of all the coordinates. As a consequence, the eigenfunctions of the Hamiltonian $\hat{H}_{0}$ depend on the coordinate $\vec{R}$ in the same way as for a free particle:

$$
\begin{aligned}
& \Phi\left(\vec{R},\left\{\vec{\rho}_{a}\right\}\right)=\frac{1}{(2 \pi)^{3 / 2}} e^{i \vec{P} \vec{R}}\left|\Phi_{1}\left(\vec{P},\left\{\vec{\rho}_{a}\right\}\right)\right\rangle, \\
& \hat{H}_{0} \rightarrow \hat{H}_{0}(\vec{P})=\sum_{a=1}^{N}\left\{\vec{\alpha}_{a}\left[\frac{1}{N} \vec{P}+\hat{\vec{p}}_{a}^{\prime}\right]+\beta_{a} m_{0}+e_{0} \hat{\varphi}\left(\vec{\rho}_{a}\right)\right\}-\frac{1}{2} \int d \vec{r}(\vec{\nabla} \hat{\varphi}(\vec{r}))^{2} .
\end{aligned}
$$


Further calculations consist in returning to the field representation by the variables $\vec{\rho}_{a}$ in the limit $(N \rightarrow \infty)$ and in using the approximate trial wave packet $\left|\Phi_{1}(\vec{P})\right\rangle$ similar to (3) but with the coefficient functions depending on $\vec{P}$. Thus, in the framework of non-perturbation QED the orthogonal and normalized set of states for the one-particle excitation of the electron-positron field is defined as follows:

$$
\left|\Phi_{1}^{(0)}(\vec{P})\right\rangle \simeq \frac{1}{(2 \pi)^{3 / 2}} e^{i \vec{P} \vec{R}} \int d \vec{q}\left\{U_{\vec{q} s}(\vec{P}) a_{\vec{q} s}^{+}+V_{\vec{q} s}(\vec{P}) b_{\vec{q} s}^{+}\right\} e^{i \vec{q} \cdot \vec{\rho}}|0 ; 0 ; \varphi(\vec{\rho})\rangle, \quad \vec{r}=\vec{R}+\vec{\rho}
$$

with the norm (46) and the coefficient functions $U_{\vec{q} s}(\vec{P}), V_{\vec{q} s}(\vec{P})$.

Using the coordinate representation for these functions

$$
\Psi_{\nu}(\vec{r}, \vec{P})=\int \frac{d \vec{q}}{(2 \pi)^{3 / 2}} \sum_{s} U_{\vec{q} s}(\vec{P}) u_{\vec{q} s \nu} e^{i \vec{q} \vec{r}}, \quad \Psi_{\nu}^{c}(\vec{r})=\int \frac{d \vec{q}}{(2 \pi)^{3 / 2}} \sum_{s} V_{\vec{q} s}(\vec{P}) v_{\vec{q} s \nu} e^{i \vec{q} \vec{r}},
$$

one can find the following functional for calculating the value $E(\vec{P})$ corresponding to the energy of one-particle excitation with an arbitrary momentum

$$
\begin{aligned}
& E(\vec{P})=\int d \vec{r}\left\{\Psi^{+}(\vec{r}, \vec{P})\left[\left(\vec{\alpha} \vec{P}-i \vec{\alpha} \vec{\nabla}+\beta m_{0}\right)+e_{0} \frac{1}{2} \varphi(\vec{r}, \vec{P})\right] \Psi(\vec{r}, \vec{P})\right. \\
& \left.-\Psi^{+c}(\vec{r}, \vec{P})\left[\left(-\vec{\alpha} \vec{P}-i \vec{\alpha} \vec{\nabla}+\beta m_{0}\right)+e_{0} \frac{1}{2} \varphi(\vec{r}, \vec{P})\right]\right\} \Psi^{c}(\vec{r}, \vec{P}), \\
& \varphi(\vec{r}, \vec{P})=e_{0} \int \frac{d \vec{r}^{\prime}}{\left|\vec{r}-\vec{r}^{\prime}\right|}\left[\Psi^{+}\left(\vec{r}^{\prime}, \vec{P}\right) \Psi\left(\vec{r}^{\prime}, \vec{P}\right)-\Psi^{+c}\left(\vec{r}^{\prime}, \vec{P}\right) \Psi^{c}\left(\vec{r}^{\prime}, \vec{P}\right)\right], \\
& \int d \vec{r}\left[\Psi^{+}(\vec{r}, \vec{P}) \Psi(\vec{r}, \vec{P})+\Psi^{+c}\left(\vec{r}^{\prime}, \vec{P}\right) \Psi^{c}\left(\vec{r}^{\prime}, \vec{P}\right)\right]=1 .
\end{aligned}
$$

It should be noted that the mean value of the operator $\hat{H}_{0}(\vec{P})$ in the representation of the second quantization was calculated by taking into account the expression

$$
\lim _{N \rightarrow \infty} \frac{1}{N} \sum_{\vec{p}}=1,
$$

that follows from a well known formula for the density of states when EPF is quantized within the normalized volume $\Omega[2]$.

It was mentioned above, that there is quite a close analogy between the nonperturbative description of QED and the theory of strong coupling in the "polaron" problem. Therefore, it is of interest to compare the obtained functional (49) with the results of various methods of including the translational motion in the "polaron" problem. The simplest one was used by Landau and Pekar [35] who introduced the Lagrange multipliers in the form

$$
J(\vec{P})=J(\vec{P}=0)+(\vec{P} \vec{V}),
$$

with the functional $J(\vec{P}=0)$ referring to a resting "polaron" and the Lagrange multiplier $V_{i}$ denoting the components of the quasi-particle average velocity.

We can see that the obtained functional (49) has the same form as (50) if the relativistic velocity of the excitation is determined by the formula

$$
\vec{V}=\int d \vec{r}\left\{\Psi^{+}(\vec{r}, \vec{P})(\vec{\alpha}) \Psi(\vec{r}, \vec{P})+\Psi^{+c}(\vec{r}, \vec{P})(\vec{\alpha}) \Psi^{c}(\vec{r}, \vec{P})\right\}
$$

corresponding to the well known interpretation of Dirac matrices [2]. 
Varying this functional by taking into account the normalized conditions leads to the following equations for the wave functions

$$
\begin{aligned}
& \left\{\left(\vec{\alpha} \vec{P}-i \vec{\alpha} \vec{\nabla}+\beta m_{0}\right)+e_{0} \varphi(\vec{r}, \vec{P})-E(\vec{P})\right\} \Psi(\vec{r}, \vec{P})=0, \\
& \left\{\left(\vec{\alpha} \vec{P}+i \vec{\alpha} \vec{\nabla}-\beta m_{0}\right)-e_{0} \varphi(\vec{r}, \vec{P})-E(\vec{P})\right\} \Psi^{c}(\vec{r}, \vec{P})=0 .
\end{aligned}
$$

These equations show that unlike the "polaron" problem [14], the translational motion of the quasi-particle determining the momentum $\vec{P}$ is related in our case to its internal movement described by the coordinate $\vec{r}$ by means of spinor variables only. The physical reason for this separation of variables is explained by the fact that in QED the self-localized state is formed by the scalar field, and its interaction with the particle does not involve the momentum exchange. In order to find the analytical energy spectrum $E(\vec{P})$ the system of non-linear equations (51) should be diagonalized with respect to to the spinor variables. The possibility of such diagonalization seems to be a non-trivial requirement for the nonperturbative QED under consideration.

The solution of the equations (51) can be found on the basis of the states for which the dependence on the vector $\vec{q}$ in the wave packet amplitudes $U_{\vec{q}, s}, V_{\vec{q}, s}$ remains the same as it was in the motionless "physical" electron. However, the relation between the spinor components of these functions can be changed. But as the self-consistent scalar potential involves the summation over all spinor components it does not depend on the momentum for the class of states in question:

$$
\varphi(\vec{r}, \vec{P})=\left.\varphi(r)\right|_{\vec{P}=0} .
$$

In the coordinate representation the transformation of the spinor components of the wave functions satisfying the equations (51) takes place because of the dependence on the momentum. The solution can be constructed by sorting out various linear combinations of the wave functions $\Psi(\vec{r}), \Psi^{c}(\vec{r}), \tilde{\Psi}(\vec{r}), \tilde{\Psi}^{c}(\vec{r})$ found in Section 2 for a resting electron. These functions correspond to the degenerated states in the case of $\vec{P}=0$ but are mixed for a moving electron. It is found that there is only one normalized linear combination satisfying all the necessary conditions of self-consistency:

$$
\begin{aligned}
& \Psi(\vec{r}, \vec{P})=L(\vec{P}) \Psi(\vec{r})+K(\vec{P}) \tilde{\Psi}(\vec{r}), \quad \Psi^{c}(\vec{r}, \vec{P})=L_{1}(\vec{P}) \Psi^{c}(\vec{r})+K_{1}(\vec{P}) \tilde{\Psi}(\vec{r}), \\
& |L|^{2}+|K|^{2}=\left|L_{1}\right|^{2}+\left|K_{1}\right|^{2}=1 .
\end{aligned}
$$

The condition (52), according to which the potential does not depend on the momentum for the excitation at the energy $E(\vec{P})$, is fulfilled if the coefficients are related as

$$
L_{1}=-K, \quad K_{1}=L .
$$

These relations are also consistent with equations (51) for wave functions.

This means that the "physical" electron moves in such a way that its states are transformed in the phase space of the orthogonal wave functions (16)-(24) but the amplitudes of its "internal" charge distributions are not changed. The analogous approach is considered for Lorentz invariant transformation of the bispinors corresponding to the free particles in QED [37].

Substituting the superpositions (53) into equations (51) we use the following relations:

$$
\begin{aligned}
& (\vec{\alpha} \vec{P}) \Psi=\left(\begin{array}{c}
i f(r)(\vec{\sigma} \vec{P}) \Omega_{1 / 2,1, M} \\
g(r)(\vec{\sigma} \vec{P}) \Omega_{1 / 2,0, M}
\end{array}\right), \quad(\vec{\alpha} \vec{P}) \Psi^{c}=\left(\begin{array}{c}
g_{1}(r)(\vec{\sigma} \vec{P}) \Omega_{1 / 2,1, M} \\
-i f_{1}(r)(\vec{\sigma} \vec{P}) \Omega_{1 / 2,0, M}
\end{array}\right), \\
& \left(-i \vec{\alpha} \vec{\nabla}+\beta m_{0}+e_{0} \varphi\right) \Psi=E(0)\left(\begin{array}{c}
g(r) \Omega_{1 / 2,0, M} \\
i f(r) \Omega_{1 / 2,1, M}
\end{array}\right), \\
& \left(-i \vec{\alpha} \vec{\nabla}+\beta m_{0}+e_{0} \varphi\right) \Psi^{c}=-E(0)\left(\begin{array}{c}
-i f_{1}(r) \Omega_{1 / 2,0, M} \\
g_{1}(r) \Omega_{1 / 2,1, M}
\end{array}\right),
\end{aligned}
$$

and similar formulas for the functions $\tilde{\Psi}(\vec{r}), \tilde{\Psi}^{c}(\vec{r}), \sigma_{i}$ are the Pauli matrices. 
For equations (51) to be fulfilled for any vector $\vec{r}$ it is necessary to set the coefficients of spherical spinors equal to the same indexes $l$. The corresponding radial functions are proved to be the same under these conditions, and the following system of equations for the spinors $\chi_{0,1}^{ \pm}$ is obtained

$$
\begin{array}{lc}
i L(\vec{\sigma} \vec{P}) \chi_{1}^{+}+K\left(E+E_{0}\right) \chi_{1}^{+}=0, & i K(\vec{\sigma} \vec{P}) \chi_{0}^{+}+L\left(E-E_{0}\right) \chi_{0}^{+}=0, \\
L(\vec{\sigma} \vec{P}) \chi_{0}^{+}+i K\left(E+E_{0}\right) \chi_{0}^{+}=0, & K(\vec{\sigma} \vec{P}) \chi_{1}^{+}+i L\left(E-E_{0}\right) \chi_{1}^{+}=0, \\
L_{1}(\vec{\sigma} \vec{P}) \chi_{1}^{-}-i K_{1}\left(E-E_{0}\right) \chi_{1}^{-}=0, & K_{1}(\vec{\sigma} \vec{P}) \chi_{0}^{-}-i L_{1}\left(E+E_{0}\right) \chi_{0}^{-}=0 \\
i L_{1}(\vec{\sigma} \vec{P}) \chi_{1}^{-}-K_{1}\left(E-E_{0}\right) \chi_{1}^{-}=0, & i K_{1}(\vec{\sigma} \vec{P}) \chi_{0}^{-}-L_{1}\left(E+E_{0}\right) \chi_{0}^{-}=0
\end{array}
$$

where the notation $E(0) \equiv E_{0}$ was used.

Spin variables in equations (54) are also separated. In order to show this one can use, for example, the relation between the coefficients resulting from the 4 th equation in (54) in the first one of these equations:

$$
\chi_{1}^{+}=i \frac{K(\vec{\sigma} \vec{P}) \chi_{1}^{+}}{L\left(E-E_{0}\right)} .
$$

As a result there exists a non-trivial solution of these equations for two branches of the energy spectrum

$$
E_{e, p}= \pm \sqrt{E_{0}^{2}+P^{2}}
$$

referring to the electron and positron zones, respectively [29]. The same expressions can be obtained for all conjugated pairs of the equations in (54). The coefficients in the wave functions (53) can be found taking into consideration the normalization condition:

$$
\begin{array}{ll}
L^{e}=K_{1}^{e}=\frac{P}{\sqrt{P^{2}+\left(E_{e}-E_{0}\right)^{2}}}, & K^{e}=-L_{1}^{e}=\frac{E_{e}-E_{0}}{\sqrt{P^{2}+\left(E_{e}-E_{0}\right)^{2}}}, \\
L^{p}=K_{1}^{p}=\frac{P}{\sqrt{P^{2}+\left(E_{e}+E_{0}\right)^{2}}}, & K^{p}=-L_{1}^{p}=-\frac{E_{e}+E_{0}}{\sqrt{P^{2}+\left(E_{e}+E_{0}\right)^{2}}} .
\end{array}
$$

One can see that this set of coefficients coincides with the set of spinor components for solving the Dirac equation for a free electron with the observed mass $m=E_{0}$ [37]. Thus, the results of this section show that the "internal" structure of the "physical" electron (positron) considered in this paper is consistent with the energy dispersion (55) for a real free particle due to the relativistic invariance of the Dirac equation.

For the interpretation of the wave packet (47) as the state vector for a "physical" particle it is essential that it is the eigenvector of the total momentum of the electron-positron field in the framework of the considered zeroth approximation. It means that the "physical" electrons with different momenta form the set of orthogonal and normalized functions if the condition (46) is taken into account:

$$
\left\langle\left\langle\Phi_{1}^{(0)}\left(\vec{P}_{1}\right) \mid \Phi_{1}^{(0)}(\vec{P})\right\rangle\right\rangle=\delta\left(\vec{P}-\vec{P}_{1}\right) .
$$

\section{Perturbation theory for QED with the "physical" electron-positron field. Discussion}

It is well known that the renormalizability is one of the most important features of QED and it is confirmed by the coincidence of its results with the experimental data. Actually it means that 
the calculated characteristics of the real electromagnetic processes do not depend on the initial parameters $e_{0}, m_{0}$ but only on the observed values of $e$ and $m$. So, we should clearly show that the effects of the nonperturbative QED refer to the internal structure of the "physical" electron (positron) only. However, its interaction with real electromagnetic field should be defined by the standard perturbation theory with the fine structure constant $\alpha \ll 1$.

Besides, it should be demonstrated that the integrals which define the corrections to the zero approximation in the perturbation theory with charge $e \sim e_{0}^{-1}$ are converged without introducing any additional regularizating parameters including the cut-off momentum.

To solve these problems, it is necessary to consider the form of the perturbation theory which uses the basis of states corresponding to the "physical" electron (positron) with various momentum. Taking into account the results of the last section let us represent the QED Hamiltonian (2) in the following form:

$$
\begin{aligned}
\hat{H}= & \hat{H}_{0}+\hat{H}_{I}, \\
\hat{H}_{0}= & \int d \vec{r}\left\{: \hat{\psi}^{*}(\vec{r})\left[-i \vec{\alpha}\left(\vec{\nabla}_{\vec{R}}+\vec{\nabla}_{\vec{r}}\right)+\beta m_{0}\right] \hat{\psi}(\vec{r}):+e_{0} \hat{\varphi}(\vec{r}): \hat{\rho}(\vec{r}):-\frac{1}{2}(\vec{\nabla} \hat{\varphi}(\vec{r}))^{2}\right\} \\
& +\sum_{\vec{k} \lambda} \omega(\vec{k}) \hat{n}_{\vec{k} \lambda}, \\
\hat{H}_{I}= & e_{0} \int d \vec{r}: \hat{\psi}^{*}(\vec{r})[\vec{\alpha} \hat{\vec{A}}(\vec{r}+\vec{R})] \hat{\psi}(\vec{r}): .
\end{aligned}
$$

The fact that the Hamiltonian depends on the coordinate $\vec{R}$ canonically conjugated to the total momentum of the electron-positron field determines the spontaneous breaking of symmetry of the system. This situation was discussed by Bogoliubov [27] for a lot of concrete physical systems. However, the global symmetry of the system is reconstructed when the observed values are averaged by the coordinate $\vec{R}$ in accordance with the norm (46).

In the previous sections the spectrum of the one-particle excitations of the electron-positron field was calculated approximately in the leading order in a power series of $\alpha_{0}^{-1}$. Let us now consider the matrix elements of the perturbation operator $H_{I}$ that are defined by the transitions between the states corresponding to the "physical" electrons (positrons) with the momenta $\vec{P}$ and $\vec{P}_{1}$ and with emission (absorption) a quanta of the transversal electromagnetic field. It is important that the vacuum state of the "physical" electron-field is also renormalized with respect to to the "primary" vacuum of the "bare" particles. Actually this new vacuum is the wave function of the collective quasi-particle states localized in the vicinity of various coordinates $\vec{R}$.

Let us write the obvious form of the initial $|i\rangle$ and final $|f\rangle$ states of this process using the definition (47) for the one-particle wave packet and the state vector of the free electromagnetic field corresponding to $N$ quanta with the wave vector $\vec{k}$ and polarization $\lambda$ :

$$
\begin{aligned}
|i\rangle= & \left|\Phi_{1}(\vec{P}), \Phi_{1}(0) N_{\vec{k}, \lambda}^{(i)}\right\rangle=\frac{1}{(2 \pi)^{3 / 2}} e^{i \vec{P} \vec{R}} \int d \vec{q}\left\{U_{\vec{q}, s}(\vec{P}) a_{\vec{q} s}^{+}+V_{\vec{q}, s}(\vec{P}) b_{\vec{q} s}^{+}\right\} e^{i \vec{q} \cdot(\vec{r}-\vec{R})} \\
& \times \frac{1}{(2 \pi)^{3 / 2}} \int d \vec{q}\left\{U_{\vec{q}, s}(0) a_{\vec{q} s}^{+}+V_{\vec{q}, s}(0) b_{\vec{q} s}^{+}\right\} e^{i \vec{q} \cdot\left(\vec{r}-\vec{R}_{1}\right)} \frac{\left(c_{\vec{k}, \lambda}^{+}\right)^{N_{\vec{k}, \lambda}^{(i)}}}{\sqrt{N_{\vec{k}, \lambda}^{(i)} !}}\left|0 ; 0 ; \varphi(\vec{r}-\vec{R}) ; \varphi\left(\vec{r}-\vec{R}_{1}\right)\right\rangle, \\
|f\rangle= & \left|\Phi_{1}(0), \Phi_{1}\left(\vec{P}_{1}\right) N_{\vec{k}, \lambda}^{(f)}\right\rangle=\frac{1}{(2 \pi)^{3 / 2}} e^{i \vec{P}_{1} \vec{R}_{1}} \int d \vec{q}\left\{U_{\vec{q}, s}\left(\vec{P}_{1}\right) a_{\vec{q} s}^{+}+V_{\vec{q}, s}\left(\vec{P}_{1}\right) b_{\vec{q} s}^{+}\right\} e^{i \vec{q} \cdot\left(\vec{r}-\vec{R}_{1}\right)} \\
& \times \frac{1}{(2 \pi)^{3 / 2}} \int d \vec{q}\left\{U_{\vec{q}, s}(0) a_{\vec{q} s}^{+}+V_{\vec{q}, s}(0) b_{\vec{q} s}^{+}\right\} e^{i \vec{q} \cdot(\vec{r}-\vec{R})} \frac{\left(c_{\vec{k}, \lambda}^{+}\right)^{N_{\vec{k}, \lambda}^{(f)}}}{\sqrt{N_{\vec{k}, \lambda}^{(f)} !}}\left|0 ; 0 ; \varphi(\vec{r}-\vec{R}) ; \varphi\left(\vec{r}-\vec{R}_{1}\right)\right\rangle .
\end{aligned}
$$


Let us calculate the matrix element of the operator $\hat{H}_{I}$ for the transition of the "physical" electron between the states with 4-momenta $P=(\vec{P}, E)$ and $P_{1}=\left(\vec{P}_{1}, E_{1}\right)$ with the emission of one quantum of the transversal electromagnetic field with $k=(\vec{k}, \omega)$. It is supposed that both fermion and photon 4-momenta are out of the mass surface. In this case the matrix element $M_{f i}=\Gamma(P, k) \delta\left(P-P_{1}-k\right)$ defines the vertex function $\Gamma(P, k)$ in the considered representation. The norm (46) is used for calculation:

$$
\begin{aligned}
M_{f i}= & \left\langle\left\langle f\left|\hat{H}_{I}\right| i\right\rangle\right\rangle=\frac{e_{0}}{\sqrt{2 k \Omega}} \sum_{s, s^{\prime}} \sum_{\mu, \nu} \int d \vec{r} \int d \vec{R} \int d \overrightarrow{R_{1}} e^{i\left(\vec{P} \cdot \vec{R}-\vec{P}_{1} \cdot \vec{R}_{1}\right)} e^{-i \vec{k} \cdot \vec{r}} \\
& \times \int \frac{d \vec{q}}{(2 \pi)^{3 / 2}} \int \frac{d \vec{q}^{\prime}}{(2 \pi)^{3 / 2}}\left\{\left[U_{\vec{q}^{\prime}, s^{\prime}}^{*}\left(\vec{P}_{1}\right) U_{\vec{q}^{\prime}, s^{\prime}}(0) U_{\vec{q}, s}^{*}(0) U_{\vec{q}, s}(\vec{P})\right.\right. \\
& \left.-V_{\vec{q}^{\prime}, s^{\prime}}^{*}\left(\vec{P}_{1}\right) V_{\vec{q}^{\prime}, s^{\prime}}(0) V_{\vec{q}, s}^{*}(0) V_{\vec{q}_{s} s}(\vec{P})\right] \\
& \left.\times\left[u_{\vec{q} s \mu}^{*}\left(\vec{\alpha} \vec{e}^{\lambda}\right)_{\mu, \nu} u_{\vec{q} s \nu}+u_{\vec{q}^{\prime} s^{\prime} \mu}^{*}\left(\vec{\alpha} \vec{e}^{\lambda}\right)_{\mu, \nu} u_{\vec{q}^{\prime} s^{\prime} \nu}\right]\right\} e^{i(\vec{r}-\vec{R}) \cdot \vec{q}} e^{i\left(\vec{r}-\vec{R}_{1}\right) \cdot \vec{q}^{\prime}}
\end{aligned}
$$

If the coordinate representation (48) is used for the coefficient functions $U_{\vec{q}, s}, V_{\vec{q}, s}$, the result is:

$$
\begin{aligned}
M_{f i}= & \frac{e_{0}}{\sqrt{2 k \Omega}} \int d \vec{r} \int d \vec{R} \int d \overrightarrow{R_{1}}\left\{\left[\Psi^{*}(\vec{r}-\vec{R}, 0)\left(\vec{\alpha} \vec{e}^{\lambda}\right) \Psi(\vec{r}-\vec{R}, \vec{P}) \Psi^{*}\left(\vec{r}-\vec{R}_{1}, \vec{P}_{1}\right) \Psi\left(\vec{r}-\vec{R}_{1}, 0\right)\right.\right. \\
& \left.+\Psi^{*}(\vec{r}-\vec{R}, 0) \Psi(\vec{r}-\vec{R}, \vec{P}) \Psi^{*}\left(\vec{r}-\vec{R}_{1}, \vec{P}_{1}\right)\left(\vec{\alpha} \vec{e}^{\lambda}\right) \Psi\left(\vec{r}-\vec{R}_{1}, 0\right)\right] \\
& -\left[\Psi^{c, *}(\vec{r}-\vec{R}, 0)\left(\vec{\alpha} \vec{e}^{\lambda}\right) \Psi^{c}(\vec{r}-\vec{R}, \vec{P}) \Psi^{c, *}\left(\vec{r}-\vec{R}_{1}, \vec{P}_{1}\right) \Psi^{c}\left(\vec{r}-\vec{R}_{1}, 0\right)\right. \\
& \left.\left.+\Psi^{c, *}(\vec{r}-\vec{R}, 0) \Psi^{c}(\vec{r}-\vec{R}, \vec{P}) \Psi^{c, *}\left(\vec{r}-\vec{R}_{1}, \vec{P}_{1}\right)\left(\vec{\alpha} \vec{e}^{\lambda}\right) \Psi^{c}\left(\vec{r}-\vec{R}_{1}, 0\right)\right]\right\} e^{i\left(\vec{P} \cdot \vec{R}-\vec{P}_{1} \cdot \vec{R}_{1}\right)} e^{-i \vec{k} \vec{r}}
\end{aligned}
$$

Let us pass to the integration over the coordinate of the center of mass $\vec{R}_{c}=\left(\vec{R}+\vec{R}_{1}\right) / 2$ and relative coordinate $\vec{R}_{0}=\left(\vec{R}-\vec{R}_{1}\right)$ in equation (57) and introduce the following vectors:

$$
\vec{r}_{1}=\vec{r}-\vec{R}_{c}-\frac{1}{2} \vec{R}_{0}, \quad \vec{r}_{2}=\vec{r}-\vec{R}_{c}+\frac{1}{2} \vec{R}_{0} .
$$

Then the vertex function results in:

$$
\begin{aligned}
M_{f i}=\Gamma( & P, k) \delta\left(\vec{P}-\vec{P}_{1}-\vec{k}\right), \quad P_{1}=P-k, \\
\Gamma(\vec{P}, \vec{k})= & \frac{e_{0}}{\sqrt{2 k \Omega}} \int d \vec{r}_{2} e^{i \vec{P}_{1} \cdot \vec{r}_{2}} \int d \vec{r}_{1} e^{-i \vec{P}_{1}}\left\{\Psi^{*}\left(r_{2}, \vec{P}_{1}\right)\left(\vec{\alpha} \vec{e}^{\lambda}\right) \Psi\left(\vec{r}_{2}, 0\right) \Psi^{*}\left(\vec{r}_{1}, 0\right) \Psi\left(\vec{r}_{1}, \vec{P}\right)\right. \\
& +\Psi^{*}\left(\vec{r}_{2}, \vec{P}_{1}\right) \Psi\left(\vec{r}_{2}, 0\right) \Psi^{*}\left(\vec{r}_{1}, 0\right)\left(\vec{\alpha} \vec{e}^{\lambda}\right) \Psi\left(\vec{r}_{1}, \vec{P}\right) \\
& -\Psi^{c, *}\left(\vec{r}_{2}, \vec{P}_{1}\right)\left(\vec{\alpha} \vec{e}^{\lambda}\right) \Psi^{c}\left(\vec{r}_{2}, 0\right) \Psi^{c, *}\left(\vec{r}_{1}, 0\right) \Psi^{c}\left(\vec{r}_{1}, \vec{P}\right) \\
& \left.-\Psi^{c, *}\left(\vec{r}_{2}, \vec{P}_{1}\right) \Psi^{c}\left(\vec{r}_{2}, 0\right) \Psi^{c, *}\left(\vec{r}_{1}, 0\right)\left(\vec{\alpha} \vec{e}^{\lambda}\right) \Psi^{c}\left(\vec{r}_{1}, \vec{P}\right)\right\} .
\end{aligned}
$$

The linear combinations (53) and the wave functions $\Psi, \Psi^{c}, \tilde{\Psi}, \tilde{\Psi}^{c}$ from equations (16), (20), (24), (25) for the resting "physical" electrons should be used in equation (58). Then one can pass to the two-component spinors in the integrand and find the following formula:

$$
\begin{aligned}
\Gamma= & i \frac{e_{0}}{\sqrt{2 k \Omega}} \int d \vec{r}_{1} \int d \vec{r}_{2}\left\{\rho ( \vec { r } _ { 1 } ) \rho ( \vec { r } _ { 2 } ) ( \chi _ { 0 } ^ { + * } ( \vec { \sigma } \vec { e } ^ { \lambda } ) \chi _ { 0 } ^ { + } ) \left[K^{*}\left(\vec{P}_{1}, E_{1}\right) L(\vec{P}, E)\right.\right. \\
& \left.-L^{*}\left(\vec{P}_{1}, E_{1}\right) K(\vec{P}, E)\right]-\rho_{1}\left(\vec{r}_{1}\right) \rho_{1}\left(\vec{r}_{2}\right)\left(\chi_{0}^{-*}\left(\vec{\sigma} \vec{e}^{\lambda}\right) \chi_{0}^{-}\right) \\
& \left.\times\left[K_{1}^{*}\left(\vec{P}_{1}, E_{1}\right) L_{1}(\vec{P}, E)-L_{1}^{*}\left(\vec{P}_{1}, E_{1}\right) K_{1}(\vec{P}, E)\right]\right\} e^{i \vec{P}_{1} \vec{r}_{1}} e^{-i \vec{P} \vec{r}}
\end{aligned}
$$

where the functions $L(\vec{P}, E), K(\vec{P}, E), L_{1}(\vec{P}, E), K_{1}(\vec{P}, E)$ are defined by equations (56) but with the energy variable out of the mass surface $E \neq E_{0}$. The charge densities are defined as follows:

$$
\rho(\vec{r})=g^{2}(r)+f^{2}(r), \quad \rho_{1}(\vec{r})=g_{1}^{2}(r)+f_{1}^{2}(r) .
$$


Accordingly to Section 2 the functions $f, g$ and $f_{1}, g_{1}$, that describe the "physical" electron internal structure, can be expressed over the universal function and are differed from each other only by the normalization conditions

$$
\begin{array}{ll}
g(r)=\frac{1}{1+C} m_{0}^{3 / 2} \frac{u_{0}\left(r m_{0}\right)}{r}, & g_{1}(r)=\frac{C}{1+C} m_{0}^{3 / 2} \frac{u_{0}\left(r m_{0}\right)}{r}, \\
f(r)=\frac{1}{1+C} m_{0}^{3 / 2} \frac{v_{0}\left(r m_{0}\right)}{\rho}, & g_{1}(r)=\frac{C}{1+C} m_{0}^{3 / 2} \frac{v_{0}\left(r m_{0}\right)}{r} .
\end{array}
$$

Besides, the spin and angular variables can be excluded by means of equations (53) for the spinor functions of the "physical" electron. As for example,

$$
K(\vec{P})\left(\vec{\sigma} \vec{e}^{\lambda}\right) \chi_{0}^{+}=\frac{i L(\vec{P})}{E+E_{0}}\left(\vec{\sigma} \vec{e}^{\lambda}\right)\left(\vec{\sigma} \vec{e}^{\lambda}\right) \chi_{0}^{+}=\frac{i L(\vec{P})}{E+E_{0}}\left(\vec{P} \vec{e}^{\lambda}\right) \chi_{0}^{+} .
$$

Taking into account the normalization conditions for the spinors $\chi_{0}^{+,-}$one can find:

$$
\begin{aligned}
\Gamma(P, k) & \left.=\frac{e_{0}}{\sqrt{2 k \Omega}} \frac{(1-C)}{(1+C)} L(\vec{P}, E) L\left(\vec{P}_{1}, E_{1}\right)\left[\frac{\left(\vec{P} \vec{e}^{\lambda}\right)}{E+E(0)}-\frac{\left(\vec{P}_{1} \vec{e}^{\lambda}\right)}{E_{1}+E(0)}\right] F^{*}(|\vec{P}-\vec{k}|) F(|\vec{P}|)\right] \\
F(|\vec{P}|) & \equiv F(\alpha,|\vec{P}| / m)=\int_{0}^{\infty} d x\left[u_{0}^{2}(x)+v_{0}^{2}(x)\right] \frac{\sin |\vec{P}| x / m_{0}}{|\vec{P}| x / m_{0}} \\
& =\int_{0}^{\infty} d x\left[u_{0}^{2}(x)+v_{0}^{2}(x)\right] \frac{\sin \alpha|\vec{P}| x / 2\left|a_{0}\right| m}{\alpha|\vec{P}| x / 2\left|a_{0}\right| m}
\end{aligned}
$$

If one use the relations $e_{0}(1-C) /(1+C)=e,(1-C) /(1+C) \sim e_{0}^{-2}, E(0)=m$ and put $F(|\vec{P}|)=1$ the expression (59) will coincide completely with the analogous matrix element in standard form of QED with the renormalized charge $e$ and mass $m$ of the electron [17]. It means that the series for the observed characteristics of the "physical" electron in a reverse power of the "primary" charge $e_{0}^{-1}$ leads to the terms with the same structure as the standard perturbation theory in a power of $e$.

However, equation (59) shows that behavior of the renormalized vertex function $\Gamma(P, k)$ at large arguments is defined by the Fourier transformation of the radial functions that describe the charge distribution in the "physical" electron. Analysis of the asymptotic behavior of the solutions of equations (28) shows that the form-factor $F(|\vec{P}|)$ at large $|\vec{P}| \gg m_{0}$ is described as follows:

$$
F(|\vec{P}|) \sim \frac{L_{0}^{4}}{\left(|\vec{P}|^{2}+L_{0}^{2}\right)^{2}}, \quad L_{0}=2 m_{0}=m \frac{4\left|a_{0}\right|}{\alpha},
$$

and tends to zero rather quickly.

So, if the representation with the basis of states corresponding to the "physical" electrons was used in the zeroth approximation then the cut-off momentum $L_{0} \sim 1 \mathrm{GeV}$ appears naturally and it has definite physical meaning. It means that in this representation the perturbation theory in a power of the observed value of the fine structure constant $\alpha \ll 1$ does not contain the ultraviolet divergencies in the integrals.

It may seem at first sight that the finite value of the cut-off momentum is in the contradiction with the experiment. It is well known that the comparison of the theoretical calculations in the framework of the standard renormalized QED with the experimental data even in the low energy range (for example, for anomalous magnetic moment) shows that no additional momentum $L \lesssim 10^{7} \mathrm{GeV}$ can be introduced in QED (for example, [33]). However, these results do not maintain that the electron is the point-like object with the form-factor equal to unity in the 
entire range of the momentum because the form-factor of the electron appears due to the high order corrections of the perturbation theory with any calculation scheme [2]. Actually it is followed from these results that the electron cannot be considered as the composite system including any parameter $L$, as for example, the hypothetical particle with a mass $M \sim L$ [33].

But the most essential feature of the present approach is the result that the cut-off momentum $L_{0}$ is not the additional parameter of the theory because it can be expressed only in terms of the observed values of QED, namely $\alpha$ and $m(!)$. The form factor of the "physical" electron in this case can be considered as the result of the partial summation of high-order corrections of usual perturbation series. Let us now show that the appearance of $L_{0}$ at the intermediate calculation stages may not contradict to the fact that it is not effect at the final results for the observed physical values in the renormalised QED.

Really, let us consider a general structure of the non-renormalized form factor of the electron as the function of the invariant parameters in the framework of QED with an arbitrary cut-off momentum $L$ and the non-renormalized values of $\alpha_{0}, m_{0}$. For definiteness, let us write only its scalar part:

$$
F_{0}(k) \equiv F_{0}\left(\alpha_{0} ; \frac{k^{2}}{m_{0}^{2}} ; \frac{m_{0}}{L}\right) .
$$

Computation of this form factor by means of the perturbation theory with the standard renormalization scheme is defined by the calculation of the vertex function [33] and corresponds to the expansion of the function (61) as a power series in both coupling constant $\alpha_{0}$ and the parameter $m_{0} / L$ :

$$
\begin{aligned}
F_{0}(k) \approx & 1+\alpha_{0}\left[F_{01}^{(0)}\left(0 ; \frac{k^{2}}{m_{0}^{2}} ; 0\right)+\frac{m_{0}}{L} F_{01}^{(1)}\left(0 ; \frac{k^{2}}{m_{0}^{2}} ; 0\right)+\cdots\right] \\
& +\alpha_{0}^{2}\left[F_{02}^{(0)}\left(0 ; \frac{k^{2}}{m_{0}^{2}} ; 0\right)+\frac{m_{0}}{L} F_{02}^{(1)}\left(0 ; \frac{k^{2}}{m_{0}^{2}} ; 0\right)+\cdots\right]+\cdots
\end{aligned}
$$

Let us remind that the QED renormalizability means that all coefficients of these series are remained finite in the limit $L \rightarrow \infty$ if the parameters $\alpha_{0}, m_{0}$ are changed by their renormalized values $\alpha, m$. Then the theoretically calculated value of the form factor is defined by the expansion in a power series of both $\alpha$ and an independent parameter $\xi \sim m / L$ :

$$
\begin{aligned}
F_{\text {th }}(k) \approx & 1+\alpha \tilde{F}_{01}^{(0)}\left(\frac{k^{2}}{m^{2}}\right)+\alpha^{2} \tilde{F}_{02}^{(0)}\left(\frac{k^{2}}{m^{2}}\right)+\cdots \\
& +\frac{m}{L}\left[\alpha \tilde{F}_{01}^{(1)}\left(\frac{k^{2}}{m^{2}}\right)+\alpha^{2} \tilde{F}_{02}^{(1)}\left(\frac{k^{2}}{m^{2}}\right)+\cdots\right]+\cdots .
\end{aligned}
$$

The formal series that appear in the intermediate stages and define the relations between the "primary" parameters of the theory $\alpha_{0}, m_{0}$ and the observed value $\alpha, m$ can be represented as follows:

$$
\begin{aligned}
& \alpha \approx \alpha_{0} A_{1}\left(\frac{m_{0}}{L}\right)+\alpha_{0}^{2} A_{2}\left(\frac{m_{0}}{L}\right)+\cdots=\alpha_{0} Z\left(\alpha_{0}, \frac{m_{0}}{L}\right), \\
& m \approx m_{0}\left[1+\alpha_{0} B_{1}\left(\frac{m_{0}}{L}\right)+\alpha_{0}^{2} B_{2}\left(\frac{m_{0}}{L}\right)+\cdots\right]=\alpha_{0} Z_{1}\left(\alpha_{0}, \frac{m_{0}}{L}\right) .
\end{aligned}
$$

Actually all coefficients of these series tend to infinity in the limit $L \rightarrow \infty$ because of the divergent integrals. Therefore the concrete values $\alpha_{0}, m_{0}$ are indefinite if usual renormalization scheme is used.

Analysis of [33] shows that in the result of the renormalization the additional terms contained the parameter $\xi$ in the expansion (63) are absent at least with an accuracy $\sim 10^{-10}$ corresponding 
to $L \lesssim 10^{7} \mathrm{GeV}$. Analogous estimation is correct when calculating characteristics of other electromagnetic processes in QED. It means that after passing to the limit $L \rightarrow \infty$ the formulas of type of equation (63) give the practical algorithm for calculation of any observed values in QED with very high accuracy. For that purpose the internal inconsistency of the theory that is conditioned by indetermination of the series (64) becomes unessential.

We believe that the variational approach presented in the paper allows one to preserve the computing possibilities of QED but to avoid its logical inconsistency. As it was shown above, in this approach the "physical" electron characteristics are defined by the quasi-particle collective excitation of the "primary" electron-positron field itself and are not conditioned by additional parameters or bond with any hypothetical particle. Therefore the perturbation series that define the corrections to the form factor conditioned by the interaction with the transverse electromagnetic field are differed from equation (62) qualitatively. According to equation (59) for the vertex function in this case the expansion parameter is the value $\alpha_{0}^{-1} \sim \alpha$ and the form factor depends on the invariant parameters as follows:

$$
F(k) \equiv F\left(\alpha_{0} ; \frac{k^{2}}{m^{2}} ; \frac{m}{m_{0}}\right), \quad \alpha_{0} \sim \alpha^{-1}, \quad m_{0} \sim m / \alpha,
$$

and the effective cut-off momentum for the divergent integrals is the value $L_{0} \sim m_{0} \sim m / \alpha$.

Then the following series arises instead of the expansion (62):

$$
\begin{aligned}
F(k) \approx & 1+\alpha_{0}^{-1}\left[F_{1}^{(0)}\left(0 ; \frac{k^{2}}{m^{2}} ; 0\right)+\frac{m}{m_{0}} F_{1}^{(1)}\left(0 ; \frac{k^{2}}{m^{2}} ; 0\right)+\cdots\right] \\
& +\alpha_{0}^{-2}\left[F_{2}^{(0)}\left(0 ; \frac{k^{2}}{m^{2}} ; 0\right)+\frac{m}{m_{0}} F_{2}^{(1)}\left(0 ; \frac{k^{2}}{m^{2}} ; 0\right)+\cdots\right]+\cdots
\end{aligned}
$$

In order to calculate the observed value for the form factor equation (65) should be completed by the series that express the "primary" parameter $\alpha_{0}, m_{0}$ over the observed values $\alpha, m$. These series have the following general form:

$$
\begin{aligned}
& \alpha \approx \alpha_{0}^{-1}\left[\lambda_{0}+\alpha_{0}^{-1} \lambda_{1}+\alpha_{0}^{-2} \lambda_{2}+\cdots\right]=\alpha_{0} \tilde{Z}\left(\alpha_{0}\right), \\
& m \approx m_{0} \alpha_{0}^{-1}\left[\mu_{0}+\alpha_{0}^{-1} \mu_{1}+\alpha_{0}^{-2} \mu_{2}+\cdots\right]=m_{0} \tilde{Z}_{1}\left(\alpha_{0}\right) .
\end{aligned}
$$

According to equation (60) all coefficients in these series are finite and and do not depend on additional parameters.

Substitution of equation (66) in equation (65) shows that the electron form factor calculated by means of the considered approach contains only the finite values and does not depend on the additional parameters as it should be in accordance with the analysis in [33]:

$$
\begin{aligned}
& \tilde{F}_{\mathrm{th}}(k) \approx 1+\alpha \tilde{F}_{1}^{(0)}\left(\frac{k^{2}}{m^{2}}\right)+\alpha^{2} \tilde{F}_{2}^{(0)}\left(\frac{k^{2}}{m^{2}}\right)+\cdots, \\
& \tilde{F}_{1}^{(0)}\left(\frac{k^{2}}{m^{2}}\right)=\frac{1}{\lambda_{0}} F_{1}^{(0)}\left(0 ; \frac{k^{2}}{m^{2}} ; 0\right), \\
& \tilde{F}_{2}^{(0)}\left(\frac{k^{2}}{m^{2}}\right)=\frac{1}{\lambda_{0}^{2}}\left[\frac{\lambda_{1}}{\lambda_{0}} F_{1}^{(0)}\left(0 ; \frac{k^{2}}{m^{2}} ; 0\right)+\frac{1}{\mu_{0}} F_{1}^{(1)}\left(0 ; \frac{k^{2}}{m^{2}} ; 0\right)+F_{2}^{(0)}\left(0 ; \frac{k^{2}}{m^{2}} ; 0\right)\right] .
\end{aligned}
$$

The considered qualitative analysis of the "physical" electron form factor can be applied not only for the interaction with the vacuum transversal electromagnetic field but also for any kind of the external fields. It means, for example, that after renormalization with the self-localized electron states the QED corrections for the "physical" electron bound in the Coulomb field of the nucleus in the hydrogen-like atom should be described by the same formulas that can be found with usual renormalization scheme [36]. 
Actually it means that the considered form of QED that uses the basis of the self-localized states and the perturbation theory on the parameter $\alpha_{0}^{-1}$ can lead to the same results for the observed values as the renormalized series in the physical fine structure constant $\alpha$. They are differed only when calculating the connections between the primary and observed values of $\alpha_{0}$ and $\alpha$ as well as $m_{0}$ and $m$. However, the strict proof of the quantitative coincidence of our results with known ones are connected with rather cumbersome calculations of high-order corrections in a power of the parameter $\alpha_{0}^{-1}$ on the basis of the vertex function (59) and the propagation function for the "physical" electron and should be considered separately.

In any case it is evident that for the practical purposes the previously existing calculation scheme in QED is more efficient than one considered in the present paper. Therefore the proof of the equivalence of the both forms of QED is important mostly from the point of the internal self-consistency of QED. Nevertheless, the suggested approach may have the applied interest as well because it allows one to consider other leptons as excitations of the "primary" electronpositron field (see Section 3 for $\mu$-meson). It is also may be essential for the constructive use of the renormalization group in QED and for the nonperturbative analysis of other quantum field models including non-renormalized ones.

\section{Conclusions}

Thus, in the present paper the self-localized quasi-particle excitation of the electron-positron field is found for the first time in the framework of a standard form of the quantum electrodynamics. Existence of a new state in the spectrum of the well investigated Hamiltonian is of great theoretical interest by itself.

Besides, the physical interpretation of the obtained solution as the "physical" electron has been considered. It allowed one to find in the finite form the relation between the charge $e_{0}$ and the mass $m_{0}$ of the "bare" electron considered as the "primary" parameters of the theory and observed values of the charge $e_{0}$ and the mass $m_{0}$ of the "physical" electron. The obtained relations between these values $\left(e_{0} \sim e^{-1}, m_{0} \sim m e^{-2}\right)$ show that they cannot be calculated by means of the standard perturbation theory.

The important consequence of the considered interpretation of the self-localized state is the possibility to represent the Dirac idea and to consider $\mu$-meson as an additional quasi-particle excitation of the electron-positron field. It is shown that the calculated mass of $\mu$-meson is very closed to its experimental value.

It is also shown that the self-localized quasi-particle state does not contradict to the Lorentzinvariance of the system and its binding energy can be considered as the observed mass of this excitation. It permitted us to describe the interaction of the "physical" electron with the transverse electromagnetic field and to show that it can considered on the basis of the perturbation theory as the series in a power of the observed charge of electron $e \sim e_{0}^{-1} \ll 1$. It proved that the coefficients of these series are free from the ultraviolet divergence.

The internal structure of the "physical" electron considered as the collective excitation does not depend on any additional parameters with the exception of $\alpha$ and $m$. It is described by the finite functions without introduction of the cut-off momentum $L$. It is also discussed quantitatively that an existence of such structure does not contradict to the experimental data about the electron form factor.

\section{Acknowledgements}

Authors are grateful to ICTP Office of External Activities for the financial support of the participation in the International Conference "Symmetry in Nonlinear Mathematical Physics" (June 24-30, 2007, Kyiv, Ukraine). 


\section{References}

[1] Weinberg S., Unified theories of elementary particle interactions, Scientific American 231 (1974), 50-58.

[2] Akhiezer A.I., Beresteckii V.B., Quantum electrodynamics, Nauka, Moscow, 1969.

Bogoliubov N.N., Shirkov D.V., Introduction to the theory of quantum fields, Nauka, Moscow, 1973.

[3] Scharf G., Finite quantum electrodynamics: the causal approach, John Wiley and Sons, Inc., USA, 2001. Scharf G., Quantum gauge theories: a true ghost story, Springer Verlag, Berlin - Heidelberg - New York, 1995.

[4] Dirac P.A.M., The principles of quantum mechanics, The Clarendon Press, Oxford, 1958.

[5] Feynman R.P., Nobel lecture, Science 153 (1966), 699-711.

[6] Lifshitz E.M., Pitaevskii L.P., Relativistic quantum theory. Part II, Nauka, Moscow, 1971.

[7] Janke W., Pelster A., Schmidt H.-J., Bachmann M. (Editors), Fluctuating paths and fields, Proceedings dedicated to Hagen Kleinert, World Scientific, Singapore, 2001.

[8] Dirac P.A.M., An extensible model of the electron, Proc. R. Soc. Lond. Ser. A Math. Phys. Eng. Sci. 268 (1962), 57-67.

[9] Klevansky S.P., The Nambu-Jona-Lasinio model of quantum chromodynamics, Rev. Modern Phys. 64 (1992), 649-671.

Gusynin V.P., Miransky V.A., Shovkovy I.A., Large $N$ dynamics in QED in a magnetic field, Phys. Rev. D 67 (2003), 107703, 4 pages, hep-ph/0304059.

[10] Hong D.K., Rajeev S.G., Towards a bosonization of quantum electrodynamics, Phys. Rev. Lett. 21 (1990), $2475-2479$.

[11] Miransky V.A., Dynamics of spontaneous chiral symmetry breaking and continuous limit in quantum electrodynamics, Nuovo Cimento A 90 (1985), 149-160.

[12] Alexandrou C., Rosenfelder R., Schreiber A.W., Nonperturbative mass renormalization in quenched QED from the worldline variational approach, Phys. Rev. D 62 (2000), 085009, 10 pages.

[13] Pekar S.I., Autolocalization of the electron in the dielectric inertially polarized media, J. Exp. Theor. Phys. 16 (1946), 335-340.

[14] Bogoliubov N.N., About one new form of the adiabatic perturbation theory in the problem of interaction between particle and quantum field, Uspekhi Mat. Nauk 2 (1950), 3-24.

Tyablikov S.V., Adiabatic form of the perturbation theory in the problem of interaction between paricle and quantum field, J. Exp. Theor. Phys. 21 (1951), 377-388.

[15] Dodd R.K., Eilbeck J.C., Gibbon J.D., Moris H.C., Solitons and nonlinear wave equations, Academic Press, London, 1984.

[16] Fröhlich H., Electrons in lattice fields, Adv. Phys. 3 (1954), 325-361.

[17] Heitler W., The quantum theory of radiation, The Clarendon Press, Oxford, 1954.

[18] Landau L.D., Lifshitz E.M., Quantum mechanics, Nauka, Moscow, 1963.

[19] Gross E.P., Strong coupling polaron theory and translational invariance, Ann. Physics, 99 (1976), 1-29.

[20] Bagan E., Lavelle M., McMullan D., Charges from dressed matter: physics and renormalization, Ann. Physics, 282 (2000), 503-540, hep-ph/9909262.

[21] Yukalov V.I., Nonperturbative theory for anharmonic oscillator, Theoret. and Math. Phys. 28 (1976), 652660.

Caswell W.E., Accurate energy levels for the anharmonic oscillator and series for the double-well potential in perturbation theory, Ann. Physics 123 (1979), 153-182.

[22] Feranchuk I.D., Komarov L.I., The operator method of the approximate solution of the Schrödinger equation, Phys. Lett. A 88 (1982), 211-214.

[23] Feranchuk I.D., Komarov L.I., Nichipor I.V., Ulyanenkov A.P., Operator method in the problem of quantum anharmonic oscillator, Ann. Physics 238 (1995), 370-440.

[24] Feranchuk I.D., Komarov L.I., Ulyanenkov A.P., Two-level system in a one-mode quantum field: numerical solution on the basis of the operator method, J. Phys. A: Math. Gen. 29 (1996), 4035-4047.

[25] Feranchuk I.D., Komarov L.I., Ulyanenkov A.P., A new method for calculation of crystal susceptibilities for x-ray diffraction at arbitrary wavelength, Acta Crystallogr. Sect. A 58 (2002), 370-384. 
[26] Fradkin E.S., Renormalization in quantum electrodynamics with self-consistent field, Proc. Fiz. Inst. of Soviet Academy of Science 29 (1965), 1-154.

Fradkin E.S., Application of functional methods in quantum field theory and quantum statistics, Nuclear Phys. 76 (1966), 588-612.

[27] Bogoliubov N.N., Quasi-mean values in the problems of statistical mechanics. Selected Works, Vol. 3, Naukova Dumka, Kiev, 1971.

[28] Komarov L.I., Krylov E.V., Feranchuk I.D., Numerical solution of the nonlinear eigenvalue problem, $Z h$. Vychisl. Mat. Mat. Fiz. 18 (1978), 681-691.

[29] Feranchuk I.D., Feranchuk S.I., Self-consistent state in the strong-coupling QED, hep-th/0309072.

[30] Bawin M., Lavine J.D., On the existence of a critical charge for superheavy nucleus, Nuovo Cimento 15 (1973), 38-44.

[31] Feranchuk I.D., Finite electron charge and mass renormalization in quantum electrodynamics, math-ph/0605028.

[32] Shnir Ya.M., Monopol, Springer, Berlin, 2005.

[33] Brodsky S.J., Drell S.D., Anomalous magnetic moment and limits on fermion substructure, Phys. Rev. D 22 (1980), 2236-2242.

[34] Feynman R.P., Slow electrons in a polar crystal, Phys. Rev. 97 (1955), 660-665.

[35] Landau L.D., Pekar S.I., Effective mass of the polaron, J. Exp. Theor. Phys. 18 (1948), 419-423.

[36] Eides M.I., Grotch H., Shelyuto V.A., Theory of light hydrogenlike atoms, Phys. Rep. 342 (2001), 63-261, hep-ph/0002158.

[37] Bjorken J.D., Drell S.D., Relativistic quantum mechanics, McGraw-Hill Book Company, London, 1976. 